Research Paper

\title{
Construction of implantation failure related IncRNA-mRNA network and identification of IncRNA biomarkers for predicting endometrial receptivity
}

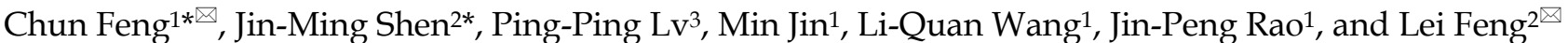

1. The Second Affiliated Hospital of Zhejiang University School of Medicine, Hangzhou, Zhejiang 310009, China.

2. The First Affiliated Hospital of Zhejiang Chinese Medicine University, Hangzhou, Zhejiang 310006, China.

3. The Women's Hospital of Zhejiang University School of Medicine, Hangzhou, Zhejiang 310006, China.

* Chun Feng and Jin-Ming Shen contributed equally to this work.

$\square$ Corresponding authors: Chun Feng, M.D., Department of Reproductive Medicine, The Second Affiliated Hospital of Zhejiang University School of Medicine, 88 Jiefang Road, Hangzhou, Zhejiang, 310009, China. Tel: +86-571-89713636. Fax: +86-571-89768001. E-mail: doctorf@@zju.edu.cn. Lei Feng, Department of Orthopedics, the First Affiliated Hospital of Zhejiang Chinese Medicine University, 54 Youdian Road, Hangzhou, Zhejiang, 310018, China. Tel: +86-571-87066752, Fax: +86-571-87071760. Email: fengleilei1982@gmail.com.

(c) Ivyspring International Publisher. This is an open access article distributed under the terms of the Creative Commons Attribution (CC BY-NC) license (https://creativecommons.org/licenses/by-nc/4.0/). See http://ivyspring.com/terms for full terms and conditions.

Received: 2018.01.22; Accepted: 2018.07.02; Published: 2018.07.27

\begin{abstract}
Insufficient endometrial receptivity is a major factor leading to implantation failure (IF), and the traditional way of morphological observation of endometrium cannot determine the condition of receptivity sufficiently. Considering that long-noncoding RNAs (IncRNAs) regulate endometrial receptivity and competing endogenous RNA (ceRNA) mechanism works in plenty of biological processes, ceRNA is likely to function in the pathology of IF. In the present study, we aim to construct an implantation failure related IncRNA-mRNA network (IFLMN), and to identify the key IncRNAs as the candidates for predicting endometrial receptivity. The global background network was constructed based on the presumed IncRNA-miRNA and miRNA-mRNA pairs obtained from IncRNASNP and miRTarBase. Differentially expressed genes (DEGs) of IF were calculated using the data of GSE26787, and then re-annotated as differentially expressed mRNAs (DEMs) and IncRNAs (DELs). IFLMN was constructed by hypergeometric test, including 255 IncRNA-mRNA pairs, 10 IncRNAs, and 212 mRNAs. Topological analysis determined the key IncRNAs with the highest centroid. Functional enrichment analyses were performed by unsupervised clustering, GO classification, KEGG pathway, and co-expression module analyses, achieving six key IncRNAs and their ceRNA sub-networks, which were involved in immunological activity, growth factor binding, vascular proliferation, apoptosis, and steroid biosynthesis in uterus and prepared endometrium for embryo implantation. Sixteen endometrial samples were collected during mid-luteal phase, including 8 recurrent implantation failure (RIF) or recurrent miscarriage (RM) women and 8 controls who conceived successfully. Quantitative real-time PCR was performed to compare the expression of the above six IncRNAs, which validated that the expression of all these IncRNAs was significantly elevated in endometrium of RIF/RM patients. Further studies are needed to investigate the underlying mechanism, and the IncRNAs may be developed into predictive biomarkers for endometrial receptivity.
\end{abstract}

Key words: Implantation failure, endometrial receptivity, long non-coding RNA (lncRNA), messenger RNA (mRNA), microRNA (miRNA), competing endogenous RNA (ceRNA), biomarkers

\section{Introduction}

Embryo viability, endometrial receptivity, and embryo-maternal cross-talk are three determinants of successful embryo implantation. Preimplantation genetic screening (PGS) is used to exclude the embryos carrying aneuploidy to facilitate pregnancy, but the clinical pregnancy rate is not as satisfying as supposed. A retrospective cohort study in 2016 analyzed 5471 cycles with PGS and found that 
transfer of euploid embryos achieved a clinical pregnancy rate around $50 \%{ }^{[1]}$. Another retrospective cohort study in US investigated 998 PGS cycles from 2011 to 2015 and found that the implantation rate was still lower than $60 \%$ after the genetic screening [2]. A study compared 88 recurrent implantation failure (IF) patients with PGS vs. without PGS, and the results showed that the clinical pregnancy rates were similar between these two groups [3]. All of these studies remind us that insufficient endometrial receptivity and failure in synchronization between embryo and endometrium is a leading cause of recurrent IF (RIF). Improving receptivity of endometrium can improve the outcome of in vitro fertilization and embryo transfer (IVF-ET). Since the traditional way to determine the condition of receptivity is simply by morphological observation of endometrium, more effective way for endometrial receptivity assessment is required.

Long non-coding RNAs (lncRNAs) are a kind of RNAs that transcript longer than 200 nt nucleotides without translated proteins. It has been widely recognized that lncRNAs have plenty of functions in various endometrial biological processes. A series of lncRNAs, such as CASC2, LINC00672, TUG1, and TUSC7, was discovered to act in endometrial cancer as tumor suppressor and promote sensitivity to chemotherapy [4-7]. Overexpression of IncRNA H19 can stimulate the steroidogenic acute regulatory protein (StAR) so as to control the ovarian steroid production ${ }^{[8]}$. Comparison of gene expression profiles of eutopic and ectopic endometrium of adenomyosis and endometriosis showed that a bundle of lncRNAs were dysregulated in ectopic endometrium [9, 10]. Furthermore, it has been reported that lncRNAs play an important role in the occurrence of IF. Animal study showed that the expression of a series of lncRNAs differed in pregnancy and non-pregnancy endometrium, which might play a vital role in facilitating embryo implantation [11]. The expression of H19 was down-regulated in the endometrium of RIF [12]. The expression of AK124742 was down-regulated together with PSMD6 in the cumulus cells of nonpregnant group compared with the pregnant group [13]. The above studies indicate that dysregulation of lncRNAs may affect the endometrial receptivity.

The competing endogenous RNA (ceRNA) theory hypothesizes that lncRNAs can regulate other RNA transcripts by competing for shared miRNAs [14]. Since the introduce of this mechanism, it has been validated that ceRNA works in the development of various tumors, and ceRNA networks has been constructed in breast cancer [15], pancreatic adenocarcinoma [16], lung cancer [17], hepatocellular carcinoma [18], glioblastoma [19], gastric cancer [20] and so on. Considering the role of lncRNAs in the regulation of endometrial receptivity and the function of ceRNA mechanism, we try to build the implantation failure related lncRNA-mRNA network (IFLMN) and to explore the key lncRNAs that can serve as biomarkers indicating endometrial receptivity.

\section{Materials and Methods}

As shown in Figure 1, we constructed the IFLMN and then analyzed the functional characteristics of IFLMN. The key lncRNAs involved in IF were identified, their related modules were investigated, the functional enrichments were studied, and at last the expression of key lncRNAs was validated in endometrium.

\section{Construction of the global IncRNA-miRNA-mRNA triple network.}

The predicted lncRNA-miRNA interact pairs were obtained from lncRNASNP2 database [21], and the predicted miRNA-mRNA interact pairs were obtained from miRTarBase [22]. Then we constructed the global triple network as the background network to identify gene interactions.

\section{Screening of differentially expressed genes (DEGs) and re-annotation.}

The gene expression data of GSE26787 was downloaded from Gene Expression Omnibus (GEO) database, which was based on the platform of GPL570 (Affymetrix Human Genome U133 Plus 2.0 Array). It compared the endometrial expression in five patients who underwent RIF after IVF/ICSI and five fertile women [23]. Data were imported into the R-studio and normalized with RMA algorithm [24]. Bioconductor limma package was applied to identify the DEGs between fertile and IF groups with fold change (FC) $>$ 2 and adjusted $P<0.05$ [25].

The DEGs were re-annotated by HG-U133 Plus_2 Annotations (CSV format, Release 36, 7/12/16) and identified as differentially expressed lncRNAs (DELs) and mRNAs (DEMs). Only the lncRNAs defined in NONCODE database were annotated as lncRNAs.

\section{Construction of the IFLMN.}

All these DELs and DEMs were mapped into the global triple network, and then lncRNA-miRNAmRNA triplets were extracted by hypergeometric test with $P<0.01$ [26], and were selected as the IncRNA-mRNA ceRNA pairs and constructed the IFLMN. 


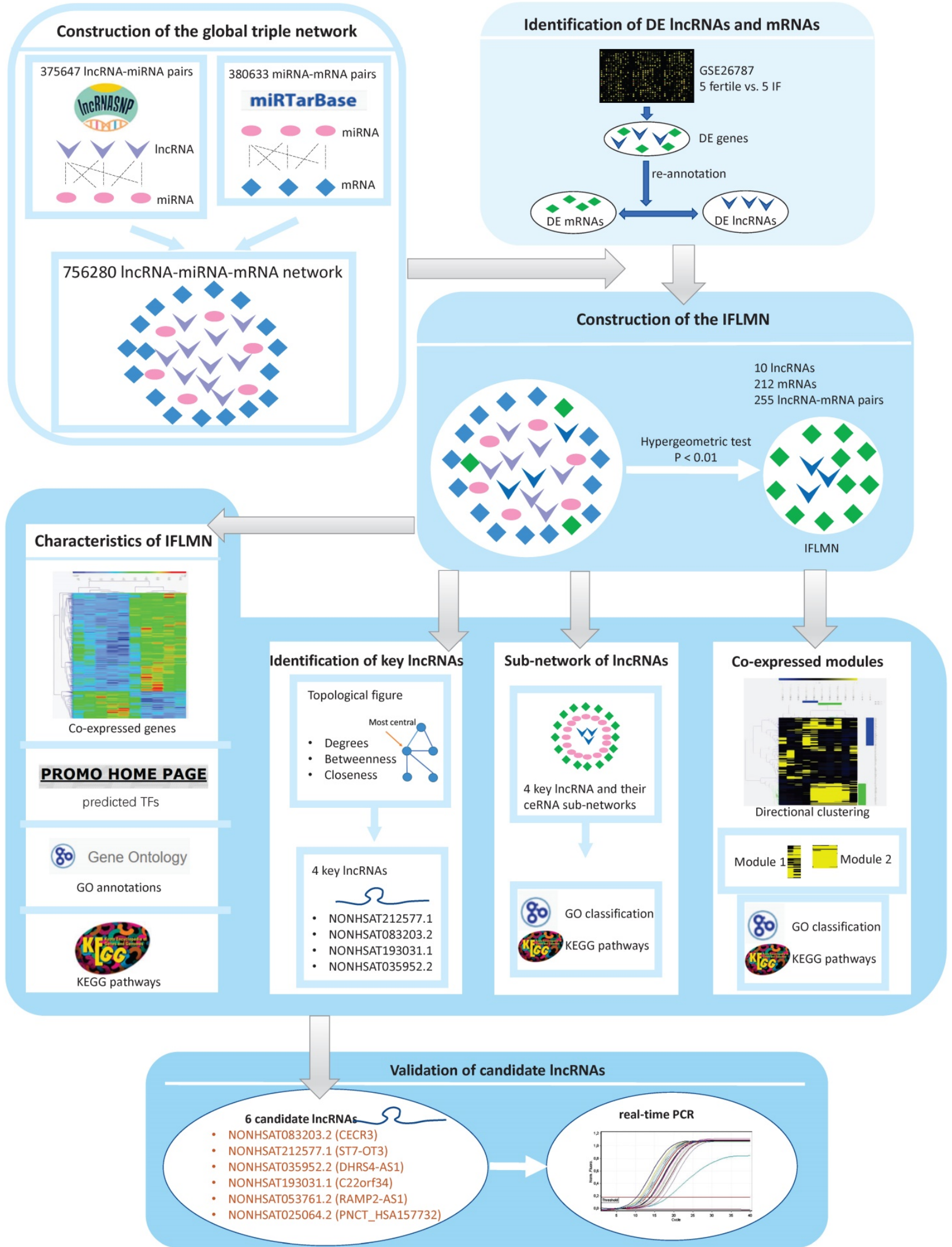

Figure 1. Workflow of the present study. First, the IncRNA-miRNA interact pairs were obtained from IncRNASNP, and the miRNA-mRNA interact pairs were obtained from miRTarBase. The pairs shared the same miRNA were combined to make up IncRNA-miRNA-mRNA triple pairs and the global triple network. Second, gene expression data were downloaded from Gene Expression Omnibus database. Differentially expressed genes (DEGs) were identified by Bioconductor limma package with fold change $>2$ and adjusted $P<0.05$. The DEGs were re-annotated and identified as differentially expressed mRNAs (DEMs) and IncRNAs (DELs). Third, IFLMN were extracted from the global network by hypergeometric test with $P<0.01$. Fourth, the functional enrichment was mined by unsupervised clustering, GO, and KEGG annotation. Fifth, the topological features of IFLMN were analyzed and four key IncRNAs were selected. Sixth, construction of the sub-network with key IncRNAs and enrichment analysis. Seventh, extracting the co-expression modules with bi-directional clustering and enrichment analysis. Eighth, validation of the expression of key IncRNAs in endometrium with real-time PCR. 


\section{Hierarchical clustering.}

Unsupervised hierarchical clustering was performed by Multiple Experiment Viewer ( $\mathrm{MeV}$ V4.9) software. The data were normalized and processed using hierarchical clustering with Pearson correlation as distance metric selection and average linkage method. The genes with similar expression profiles were grouped together and selected as the candidates for further co-expression sub-network. Hierarchical clustering was performed on IFLMN and on the lncRNA related mRNAs.

\section{Enrichment analysis, including GO and KEGG pathway analysis.}

Gene Ontology (GO) terms of biological process and molecular function were used to determine the function of target genes. Kyoto Encyclopedia of Genes and Genomes (KEGG) pathway analysis was applied to explore the significant pathways of DEGs. We performed GO classification and KEGG pathway analyses using Cytoscape V3.5.1 with the ClueGo V2.3.5 plug-in. The p-value was calculated by two-sided hypergeometric test and BenjaminiHochberg adjustment. GO terms and KEGG pathways were identified as enriched in target genes when $P \leq$ 0.05 . We analyzed all of the target genes in IFLMN and ceRNA sub-networks using GO and KEGG pathway analyses, and the results were visualized with Cytoscape.

\section{Topological analysis and selection of key genes.}

The topological analysis of DELs and DEMs was conducted to identify the central nodes of IFLMN. The topological parameters, including degree, betweenness, and closeness, were calculated by Cytoscape with CentiScaPe 2.2 plug-in. The top-ranked genes in degree, betweenness, and closeness were compared and the overlapped genes in these three topological parameters were selected as the key genes for further ceRNA analysis.

\section{Prediction of transcription factors (TFs).}

To characterize the potential association between the key lncRNAs, the TFs that might regulate the lncRNAs were identified. The sequences of presumed promoters were downloaded from UCSC genome browser database. PROMO software was used to detect the predicted TFs with Maximum matrix dissimilarity rate $<3$. The Venn diagram was used to obtain the overlapping TFs targeting all the key lncRNAs.

\section{Construction of ceRNA sub-networks.}

The key lncRNAs and its first mRNA neighbors clustered together were extracted from the IFLMN.
The miRNAs connecting the lncRNA and mRNA pairs were extracted from the global triple network and used to construct the IncRNA-miRNA-mRNA triple pairs. The ceRNA sub-networks were constructed by all these competing triplets and were visualized with Cytoscape software.

\section{Bidirectional clustering.}

The gene co-expression modules were extracted from IFLMN by bidirectional clustering. The Pearson correlation coefficient (PCC) between lncRNA and mRNA was calculated and lncRNA-mRNA pairs with strong correlations (PCC $>0.6, P<0.05$ ) were selected for further bidirectional clustering. There were 10 lncRNAs, 163 mRNAs, and 192 lncRNA-mRNA pairs involved for further bidirectional clustering. These genes were hierarchically clustered on the basis of Pearson correlation, and co-expression modules were explored by computing distances with an average linkage matrix.

\section{Sample collection}

This study was approved by the Ethics Committee of the Second Affiliated Hospital, School of Medicine, Zhejiang University. All participants signed informed written consents. Participants were recruited from the Second Affiliated Hospital of Zhejiang University School of Medicine and the Women's Hospital of Zhejiang University School of Medicine. Eight patients that failed to get pregnant after $\geq 3$ times of embryo transfers or who experienced $\geq 3$ times of pregnancy losses during 6-12 weeks were recruited in RIF group. Eight patients that conceived after their first embryo transfer were included in control group. Exclusion criteria were as follows: hysteromyoma, adenomyosis, endometrial polyps, intrauterine adhesion, endometriosis, or hydrosalpinx. Endometrial specimen was collected at mid-luteal phase with a SAP-1 device (Saipujiuzhou, Beijing, China). The specimens were put in $300 \mu 1$ RNAiso Plus and stored at $-80^{\circ} \mathrm{C}$ until use.

\section{RNA Extraction and Reverse Transcription}

Total RNA was extracted from endometrial tissue using RNAiso Plus (TaKaRa, Dalian, China, 9109) according to manufacturer's instructions. The concentration and purity of RNA were determined with spectrophotometer (Nanodrop 2000, Thermo Scientific). RNA samples were stored at $-80^{\circ} \mathrm{C}$. Total RNA with good purity and integrity was reverse transcribed using PrimeScript ${ }^{\mathrm{T}}$ RT Master Mix (TaKaRa, Dalian, China, RR036A) on the Mastercycler (Eppendorf AG 22331 Hamburg, Germany) under the following conditions: $37^{\circ} \mathrm{C}$ for 15 minutes, $85^{\circ} \mathrm{C}$ for 5 seconds, and $4^{\circ} \mathrm{C}$. cDNA samples were stored at $-20^{\circ} \mathrm{C}$. 
Table 1. Primer sequences for real-time RT-PCR

\begin{tabular}{|c|c|c|c|c|}
\hline LncRNAs & Genes & Primer forward & Primer reverse & Product size \\
\hline NONHSAT083203.2 & CECR3 & 5'-CСTCCTGGCAGAAAAGTTCCC-3' & 5'-AAGGCAGAAGGACCAGTAGG-3' & $150 \mathrm{bp}$ \\
\hline NONHSAT212577.1 & ST7-OT3 & 5'-GGCCTCTAAGAAACTTCGTGT-3' & 5'-TGTCCTTCTTCAGGCTAGGG-3' & $110 \mathrm{bp}$ \\
\hline NONHSAT035952.2 & DHRS4-AS1 & 5'-ATCGATAGGGCTGCACAGAG-3' & 5'-TGTCCTCAGACCTTACACGC-3' & $75 b p$ \\
\hline NONHSAT193031.1 & C22orf34 & 5'-ATGCTCACAGGGATGTCACG-3' & 5'-AACCCATTCCAAGCGGATCA-3' & $165 \mathrm{bp}$ \\
\hline NONHSAT053761.2 & RAMP2-AS1 & 5'-GATCTATTTCGGGGGCTCGG-3' & 5'-CGCTTCTCCTCCCTGGTTTT-3' & $144 \mathrm{bp}$ \\
\hline \multirow[t]{2}{*}{ NONHSAT025064.2 } & PNCT_HSA157732 & 5'-TAGCCAGCTGAATTGCCACA-3' & 5'-CGCTGGAGATCGTTCAGGAAA-3' & $75 b p$ \\
\hline & АCTB & 5'-CGCGAGAAGATGACCCAGAT-3' & 5'-ACAGCCTGGATAGCAACGTA-3' & $71 b p$ \\
\hline
\end{tabular}

For PCR, the primers were designed using Primer-blast (https://www.ncbi.nlm.nih.gov/tools/primer-blast). All PCR reactions were repeated in triplicate for both target genes and internal controls.

\section{Quantitative Real-Time PCR}

Real-time RT-PCR analysis was run on 7500 Fast Real-Time PCR System (Applied Biosystems) using a TB Green ${ }^{\mathrm{TM}}$ Premix Ex Taq ${ }^{\mathrm{TM}}$ II kit (TaKaRa, Dalian, China, RR820A), according to the manufacturer's protocol. The primer sequences for real-time RT-PCR were listed in Table 1 . The following parameters were used: $95^{\circ} \mathrm{C}$ for 30 seconds; 40 cycles of $95^{\circ} \mathrm{C}$ for 5 seconds and $60^{\circ} \mathrm{C}$ for 34 seconds. All the experiments were repeated in triplicate. The housekeeping gene ACTB was used as an endogenous reference. The primer sequences used in this study were listed in Table 1 . The relative mRNA expression levels were calculated using the 2- $\Delta \Delta \mathrm{Ct}$ method [27].

\section{Results and Discussion}

\section{Construction of the global IncRNA-miRNA-mRNA triple network.}

In all, there were 375647 lncRNA-miRNA interaction pairs, 19422 lncRNAs, and 2588 miRNAs obtained from the lncRNASNP2 database. 380633 miRNA-mRNA interaction pairs, 2599 miRNAs, and 15050 mRNAs were downloaded from miRTarBase. As shown in Figure S1, IncRNA-miRNA pairs and miRNA-mRNA pairs were combined and constructed the global triple network, which included 756280 edges, 19422 lncRNAs, 2599 miRNAs, and 15050 mRNAs. This global network was used as a background network to construct the IFLMN.

\section{Construction of the implantation failure related IncRNA-mRNA network.}

The gene expression data of GSE26787 was obtained from GEO database, comparing the endometrial expression between five RIF patients and five fertile women. 1251 DEGs were identified with FC $>2$ and adjusted $P<0.05$, including 990 genes up-regulated and 261 genes down-regulated in IF group. Only the lncRNAs defined in NONCODE database were annotated as IncRNA, and the DEGs were re-annotated and identified as 77 DELs and 1029 DEMs.

All these DELs and DEMs were mapped into the global triple network, and then 1750 lncRNA-miRNA-
mRNA triplets were extracted by hypergeometric test with $P<0.01$. Finally, 255 lncRNA-mRNA pairs were selected as the lncRNA-mRNA ceRNA pairs and constructed the IFLMN, including 10 lncRNAs and 212 mRNAs, as shown in Figure 2A.

\section{The functional characteristics of IFLMN.}

Enrichment analyses of GO classification and KEGG Pathway were performed on the DELs and DEMs in IFLMN. GO molecular function analysis found 55 GO terms enriched ( $P \leq 0.05$ adjusted with Benjamini-Hochberg), as shown in Table 2 and Figure 2B. The result of KEGG pathway revealed that these genes might be involved in the nine pathways listed in Table 3 and Figure $2 C(P \leq 0.05)$.

Polymeric immunoglobulin receptor activity and interferon-gamma receptor binding were found to be enriched in IFLMN, with polymeric immunoglobulin receptor (PIGR) and interferon gamma (IFNG) (Figure 2B). The recent transcriptional profile study on endometrial receptivity proved that immunological activity and vascular proliferation belong to the most over-expressed biological functions during window of implantation (WOI) [28]. The expression of molecules with immune function changes during WOI [29]. Animal study revealed that the decidual immune cell profile is consistently maintained during successive pregnancies and the decidua immune environment in pregnancy is precisely regulated [30]. IFNG is a proinflammatory cytokine secreted by uterine natural killer cells in maternal endometrium and by trophoblasts during early pregnancy. IFNG plays critical roles in initiating endometrial vasculature remodeling and angiogenesis at implantation sites [31, 32]. Meanwhile, peptide-transporting ATPase activity was enriched in IFLMN, including TAP1 and TAP2. IFNG induces transcription of antigen peptide transporter (TAP1 and TAP2) and involve in the preparation for implantation [32, 33].

Growth factor binding was enriched in IFLMN, including KAZALD1, NGFR, HAP1, ITGB3, NTRK3, and PCSK6 (Figure 2B). Endometrial receptivity is affected by the interaction between embryo and endometrium via cytokines, growth factors, 
hormones and so on [34]. A series of growth factors, such as insulin like growth factor (IGF) [35], vascular endothelial growth factor (VEGF) [36, 37], transforming growth factor (TGF) [38], are involved in the endometrial receptivity. IGF1R expression decreases after ormeloxifene treatment and inhibits embryo implantation [35].
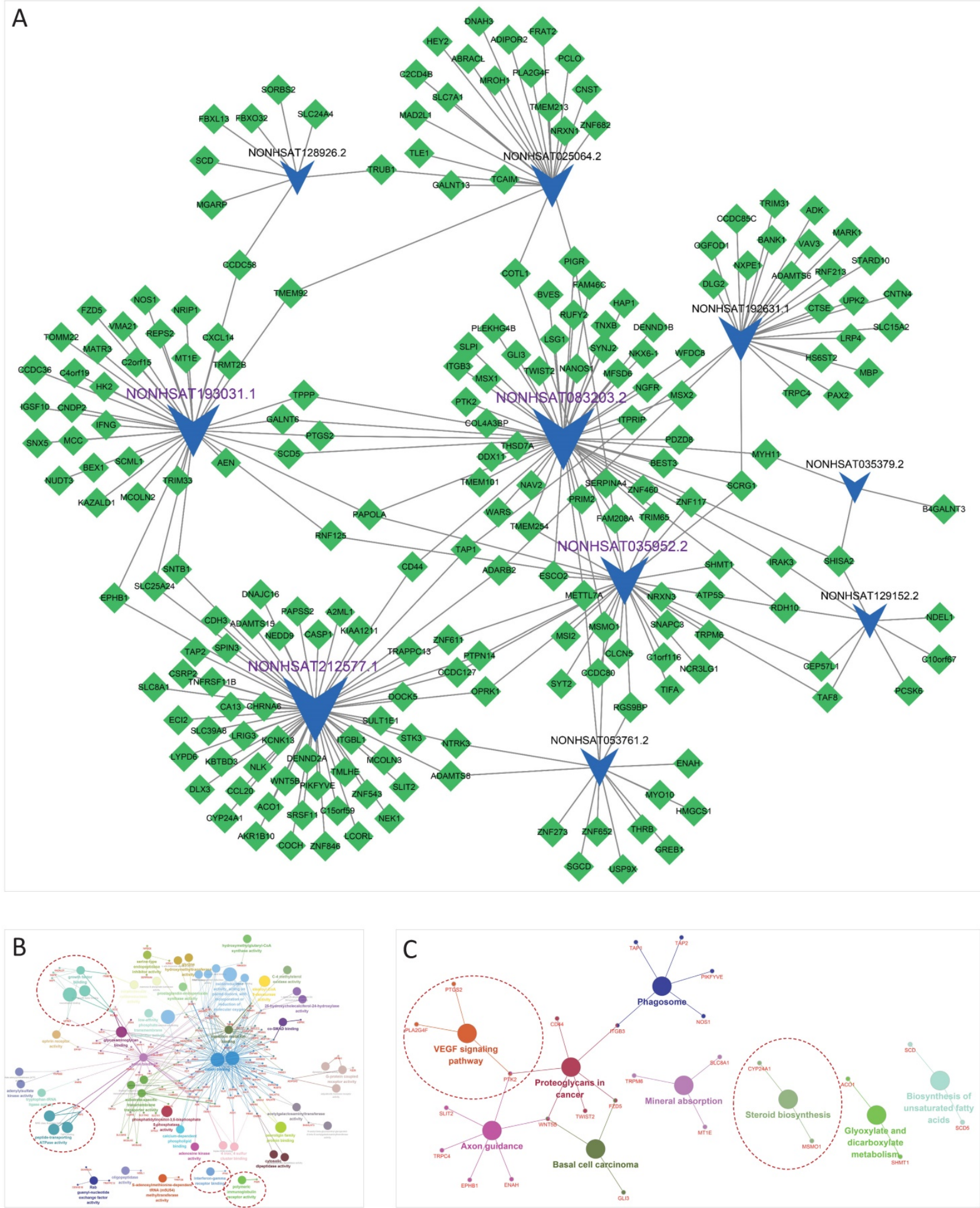

Figure 2. Implantation failure related IncRNA-mRNA network (IFLMN) and enrichment analysis. A. The blue concave quadrilateral represents IncRNA and the green diamond represents mRNA. Grey edges indicate IncRNA-mRNA interactions. Size of the node represents the degrees of the nodes in IFLMN. The IncRNAs marked with purple labels are the four most central IncRNAs. B. GO analysis of IFLMN. C. KEGG pathway analysis of IFLMN. The dotted lines emphasize the annotations that have been proved to work in endometrial receptivity. 
Table 2. Enriched GO terms in IFLMN.

\begin{tabular}{|c|c|c|}
\hline GO Term & P-Value & Enrichment score \\
\hline C-4 methylsterol oxidase activity & $2.14 \mathrm{E}-02$ & 100.00 \\
\hline polymeric immunoglobulin receptor activity & 2.14E-02 & 100.00 \\
\hline low-affinity phosphate transmembrane transporter activity & $2.14 \mathrm{E}-02$ & 100.00 \\
\hline adenosine kinase activity & $2.14 \mathrm{E}-02$ & 100.00 \\
\hline S-adenosylmethionine-dependent tRNA (m5U54) methyltransferase activity & $2.14 \mathrm{E}-02$ & 100.00 \\
\hline oligopeptidase activity & $2.14 \mathrm{E}-02$ & 100.00 \\
\hline 25-hydroxycholecalciferol-24-hydroxylase activity & 2.14E-02 & 100.00 \\
\hline 1-alpha,25-dihydroxyvitamin D3 24-hydroxylase activity & $2.14 \mathrm{E}-02$ & 100.00 \\
\hline triplex DNA binding & $2.14 \mathrm{E}-02$ & 100.00 \\
\hline cytosolic dipeptidase activity & $2.14 \mathrm{E}-02$ & 100.00 \\
\hline alanylglutamate dipeptidase activity & $2.14 \mathrm{E}-02$ & 100.00 \\
\hline dynorphin receptor activity & $2.14 \mathrm{E}-02$ & 100.00 \\
\hline stearoyl-CoA 9-desaturase activity & $6.78 \mathrm{E}-04$ & 66.67 \\
\hline tryptophan-tRNA ligase activity & $4.26 \mathrm{E}-02$ & 50.00 \\
\hline hydroxymethylglutaryl-CoA synthase activity & 4.26E-02 & 50.00 \\
\hline prostaglandin-endoperoxide synthase activity & $4.26 \mathrm{E}-02$ & 50.00 \\
\hline interferon-gamma receptor binding & 4.26E-02 & 50.00 \\
\hline glycine hydroxymethyltransferase activity & $4.26 \mathrm{E}-02$ & 50.00 \\
\hline L-allo-threonine aldolase activity & $4.26 \mathrm{E}-02$ & 50.00 \\
\hline mannose-6-phosphate isomerase activity & $4.26 \mathrm{E}-02$ & 50.00 \\
\hline $\mathrm{N}$-acetyl-beta-glucosaminyl-glycoprotein 4-beta- $\mathrm{N}$-acetylgalactosaminyltransferase activity & $4.26 \mathrm{E}-02$ & 50.00 \\
\hline adenylylsulfate kinase activity & $4.26 \mathrm{E}-02$ & 50.00 \\
\hline sulfate adenylyltransferase (ATP) activity & $4.26 \mathrm{E}-02$ & 50.00 \\
\hline adipokinetic hormone receptor activity & $4.26 \mathrm{E}-02$ & 50.00 \\
\hline phosphatidylinositol-3,5-bisphosphate 5-phosphatase activity & $2.23 \mathrm{E}-03$ & 40.00 \\
\hline neurotrophin binding & 7.89E-06 & 36.36 \\
\hline neuroligin family protein binding & 3.32E-03 & 33.33 \\
\hline TAP1 binding & 3.32E-03 & 33.33 \\
\hline MHC class Ib protein binding & 3.32E-03 & 33.33 \\
\hline peptide-transporting ATPase activity & $3.32 \mathrm{E}-03$ & 33.33 \\
\hline nerve growth factor binding & 3.32E-03 & 33.33 \\
\hline cadmium ion binding & 1.93E-04 & 33.33 \\
\hline NADPH-hemoprotein reductase activity & $9.68 \mathrm{E}-03$ & 20.00 \\
\hline calcium:cation antiporter activity & $1.40 \mathrm{E}-02$ & 16.67 \\
\hline co-SMAD binding & 1.64E-02 & 15.38 \\
\hline ephrin receptor activity & $3.45 \mathrm{E}-02$ & 10.53 \\
\hline FMN binding & 3.81E-02 & 10.00 \\
\hline acetylgalactosaminyltransferase activity & 1.23E-02 & 8.57 \\
\hline 4 iron, 4 sulfur cluster binding & 2.31E-02 & 6.82 \\
\hline calcium-dependent phospholipid binding & 3.09E-02 & 6.12 \\
\hline Rab guanyl-nucleotide exchange factor activity & 4.01E-02 & 5.56 \\
\hline intramolecular oxidoreductase activity & $4.21 \mathrm{E}-02$ & 5.45 \\
\hline oxidoreductase activity, acting on paired donors, with incorporation or reduction of molecular oxygen & 2.07E-04 & 5.20 \\
\hline growth factor binding & 6.89E-03 & 4.41 \\
\hline serine-type endopeptidase inhibitor activity & 4.49E-02 & 4.00 \\
\hline iron ion binding & $5.78 \mathrm{E}-03$ & 3.98 \\
\hline divalent inorganic cation transmembrane transporter activity & 7.17E-03 & 3.83 \\
\hline glycosaminoglycan binding & 4.47E-03 & 3.72 \\
\hline organic acid binding & 4.06E-02 & 3.02 \\
\hline substrate-specific transmembrane transporter activity & $3.06 \mathrm{E}-02$ & 1.87 \\
\hline transition metal ion binding & 7.56E-03 & 1.84 \\
\hline cation binding & 3.21E-05 & 1.66 \\
\hline metal ion binding & $5.65 \mathrm{E}-05$ & 1.66 \\
\hline anion binding & $8.11 \mathrm{E}-03$ & 1.57 \\
\hline G-protein coupled receptor activity & 2.19E-02 & 0.33 \\
\hline
\end{tabular}

Table 3. Enriched KEGG Pathways in IFLMN.

\begin{tabular}{lll}
\hline GO Term & P-Value & Enrichment score \\
\hline Steroid biosynthesis & $2.39 \mathrm{E}-02$ & 10.00 \\
Biosynthesis of unsaturated fatty acids & $3.10 \mathrm{E}-02$ & 8.70 \\
Glyoxylate and dicarboxylate metabolism & $4.46 \mathrm{E}-02$ & 7.14 \\
Mineral absorption & $2.47 \mathrm{E}-02$ & 5.77 \\
Basal cell carcinoma & $2.85 \mathrm{E}-02$ & 5.45 \\
VEGF signaling pathway & $3.42 \mathrm{E}-02$ & 5.08 \\
Axon guidance & $1.89 \mathrm{E}-02$ & 3.43 \\
Phagosome & $3.83 \mathrm{E}-02$ & 3.25 \\
Proteoglycans in cancer & $3.58 \mathrm{E}-02$ & 2.96 \\
\hline
\end{tabular}


Steroid biosynthesis pathway was enriched in IFLMN (Figure 2C). It is reported that decidualization of endometrial stromal cells is characterized by an increase in aromatase activity and activates the biosynthesis and metabolism of estrogen [39]. Estrogen plays a critical role in regulating endometrial natural killer and endothelial cells within the endometrium during early pregnancy [39]. The variation of steroid biosynthesis pathway may affect the regional concentration of steroid in endometrium and disturb the environment of embryo implantation [40]. These results indicate that endometrial steroid biosynthesis plays a pivotal role in the establishment of a receptive endometrium environment.

VEGF signaling pathway was enriched in IFLMN (Figure 2C). VEGF is expressed in the endometrium and changes cyclically [41]. It is involved in angiogenesis, an important phenomenon during embryo implantation [42, 43]. During IVF/ICSI, VEGF expression is significantly higher in the women that became pregnant compared with those nonpregnant [44]. The increased progesterone level may affect the VEGF angiogenic factors of endometrium and decrease endometrial receptivity [45].

\section{A co-expression module in IFLMN and the putative transfer factors.}

The 222 differentially expressed genes included in IFLMN were clustered. As shown in Figure 3A, unsupervised hierarchical clustering of fertile and IF samples indicated a unique gene expression profile between fertile and IF groups. Meanwhile, 27 genes revealed a co-expression module, including 4 DELs and 23 DEMs, with a notably up-regulated expression in IF. These four DELs were NONHSAT083203.2, NONHSAT025064.2, NONHSAT129152.2, and NONHSAT193031.1.

According to the sequences of promoters, the TFs of the four DELs were predicted on PROMO with Maximum matrix dissimilarity rate $<3$. There were 25 common TFs appeared in these four lncRNAs, as shown in Figure 3B. Among them, five TFs were involved in the sex steroid functions, and they were marked in brown in Figure 3B, including C/EBPbeta, C/EBPalpha, ERalpha, PRA, and PRB. During pregnancy progesterone exerts a profound influence on uterus via PRA and PRB [46]. The expression of CCAAT/enhancer binding protein beta (C/EBPbeta) is regulated by progesterone in stroma and by estrogen in uterine epithelium [47]. PRA and PRB collaborate with C/EBPbeta to regulate the expression of many target genes to control uterine epithelial proliferation, stromal differentiation, angiogenesis, and local immune response to render the endometrial receptivity and allow embryo implantation [46, 49].
Absence of C/EBPbeta leads to a block of stromal cell proliferation after decidual stimulation, causing a complete failure of the implantation [50]. Loss of appropriate ERalpha down-regulation in mid-secretory phase may lead to some types of uterine receptivity defects ${ }^{[51]}$. In all, sex steroids, TFs, lncRNAs and down-stream effectors constitute a network regulating endometrial receptivity and prepare endometrium for implantation.

GO biological process analysis with $P \leq 0.01$ adjusted with Benjamini-Hochberg found $13 \mathrm{GO}$ terms enriched, as shown in Figure $3 \mathrm{C}$, including positive regulation of interleukin-1 alpha biosynthetic process, elastic fiber assembly, mRNA pseudouridine synthesis, regulation of microtubule motor activity, positive regulation of axon regeneration, odontogenesis of dentin-containing tooth, odontoblast differentiation, thymocyte migration, diphosphoinositol polyphosphate catabolic process, diadenosine pentaphosphate catabolic process, diadenosine hexaphosphate catabolic process, and adenosine 5'-(hexahydrogen pentaphosphate) catabolic process. The result of KEGG pathway with $P$ $\leq 0.07$ revealed that these genes might be involved in the five pathways listed in Figure 3D, including ECM-receptor interaction, Ribosome biogenesis in eukaryotes, IL-17 signaling pathway, TNF signaling pathway, and Rheumatoid arthritis.

Positive regulation of interleukin-1 alpha biosynthetic process was enriched in this module (Figure 3C). During endometrial stromal cell decidualization there is a significant modulation of endometrial cell receptivity to IL1 family members and IL1R, which suggests the involvement of IL1 family members in physiological processes of endometrial receptivity [54, 55]. Extracellular matrix (ECM)-receptor interaction pathway was enriched in this module (Figure 3D). Both human and animal studies indicate that not only DEGs between RIF and conception after IVF/ICSI, but also DEGs between receptive endometrium and pre-receptive phase endometrium, are mainly participated in ECM-receptor interaction pathway $[56,57]$.

\section{Topological characteristics of IFLMN}

The topological characteristics of IFLMN were analyzed with degree, betweenness, and closeness. The genes in IFLMN with the highest degree, betweenness, or closeness were listed in Table 4 . There are four genes existed simultaneously on all the three top 10 lists, that is, four lncRNAs, as shown in Figure 4A. The top four genes with the highest degree coincide with these four IncRNAs, and they are NONHSAT212577.1, NONHSAT083203.2, NONHSA T193031.1, and NONHSAT035952.2 (Figure 4B). 
A

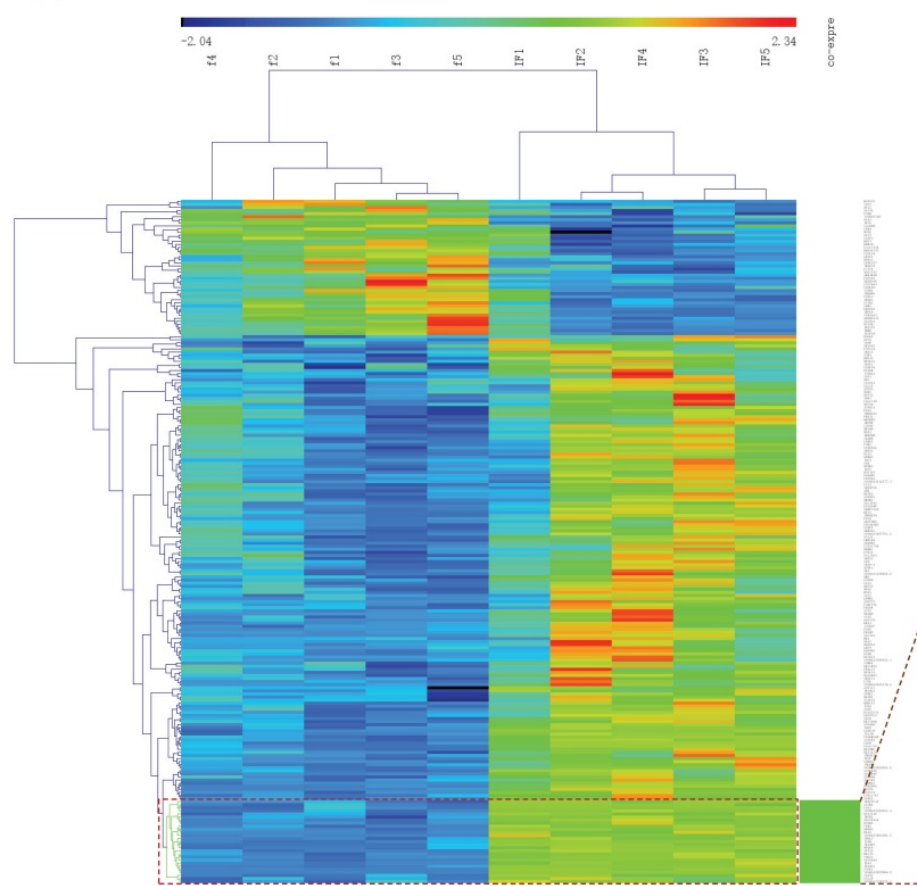

C

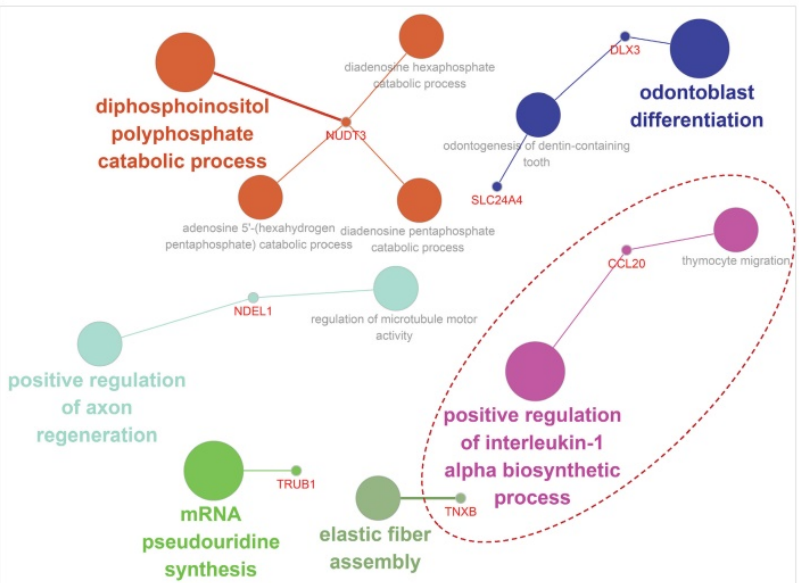

B
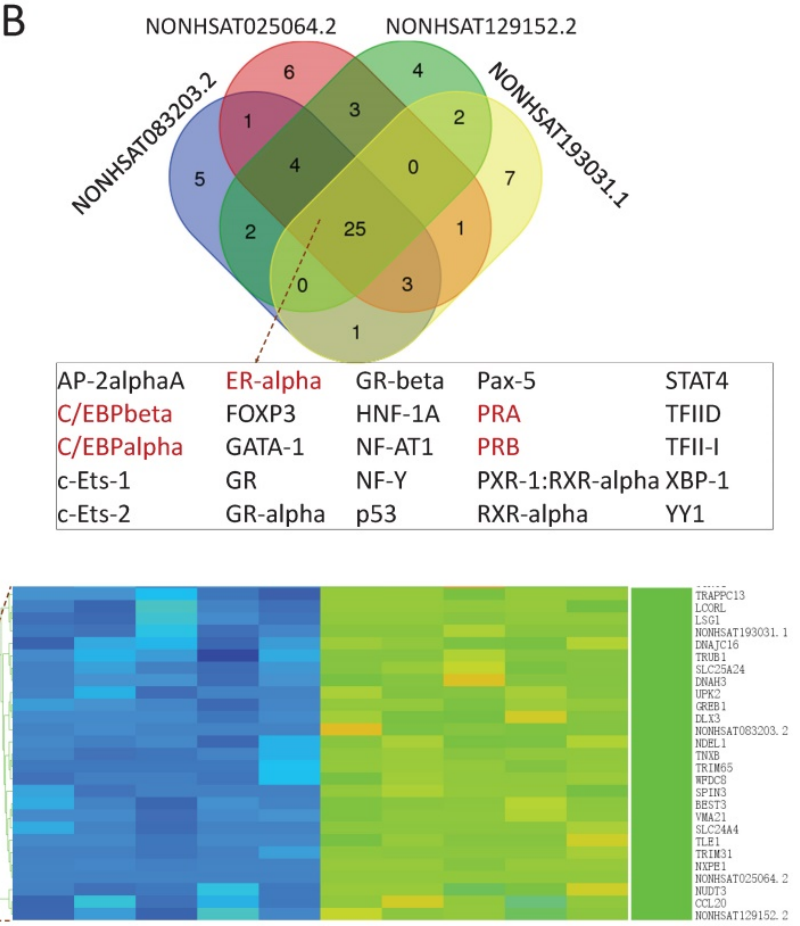

D

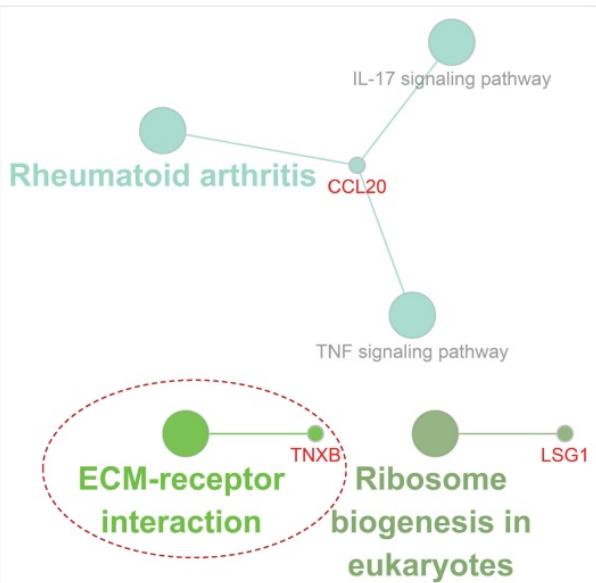

Figure 3. Custer analysis of implantation failure related IncRNA-mRNA network (IFLMN) and its co-expression network. A. The clustering of the IFLMN genes. Unsupervised clustering of IFLMN genes could discriminate between fertile and IF groups. B. The Venn of predicted transcription factors (TFs) of the four co-expression IncRNAs. There are 25 common TFs and TFs involved in the sexual hormone functions were marked in brown. C. GO enrichment analysis of co-expression mRNAs. D. KEGG pathway analysis of co-expression mRNAs. The dotted lines emphasize the annotations that have been proved to work in endometrial receptivity.

Table 4. The 10 top genes with highest degrees, betweenness, and closeness.

\begin{tabular}{|c|c|c|c|c|c|c|}
\hline $\mathbf{N}$ & Genes & Degree & Genes & Betweenness & Genes & Closeness \\
\hline 1 & NONHSAT212577.1 & 57 & NONHSAT212577.1 & 20696.65 & NONHSAT083203.2 & 0.001773 \\
\hline 2 & NONHSAT083203.2 & 52 & NONHSAT083203.2 & 19838.70 & NONHSAT212577.1 & 0.001650 \\
\hline 3 & NONHSAT193031.1 & 37 & NONHSAT193031.1 & 14863.68 & NONHSAT035952.2 & 0.001634 \\
\hline 4 & NONHSAT035952.2 & 30 & NONHSAT035952.2 & 12343.36 & NONHSAT193031.1 & 0.001524 \\
\hline 5 & NONHSAT192631.1 & 24 & NONHSAT192631.1 & 8979.926 & PAPOLA & 0.001511 \\
\hline 6 & NONHSAT025064.2 & 22 & NONHSAT025064.2 & 8438.824 & CD44 & 0.001493 \\
\hline 7 & NONHSAT053761.2 & 14 & SCRG1 & 5681.178 & PIGR & 0.001462 \\
\hline 8 & NONHSAT128926.2 & 8 & PIGR & 4668.291 & SCRG1 & 0.001445 \\
\hline 9 & NONHSAT129152.2 & 8 & CD44 & 4418.550 & EPHB1 & 0.001425 \\
\hline 10 & MSMO1 & 3 & NONHSAT053761.2 & 4236.610 & SLC25A24 & 0.001425 \\
\hline
\end{tabular}

For NONHSAT212577.1, 57 mRNAs were extracted from the IFLMN as the first mRNA neighbors. GO classification with $P \leq 0.05$ adjusted by
Benjamini-Hochberg found three enriched GO terms for Molecular function and one GO term for Biological process, as shown in Figure 4C and 4D, and Table S1. 
A
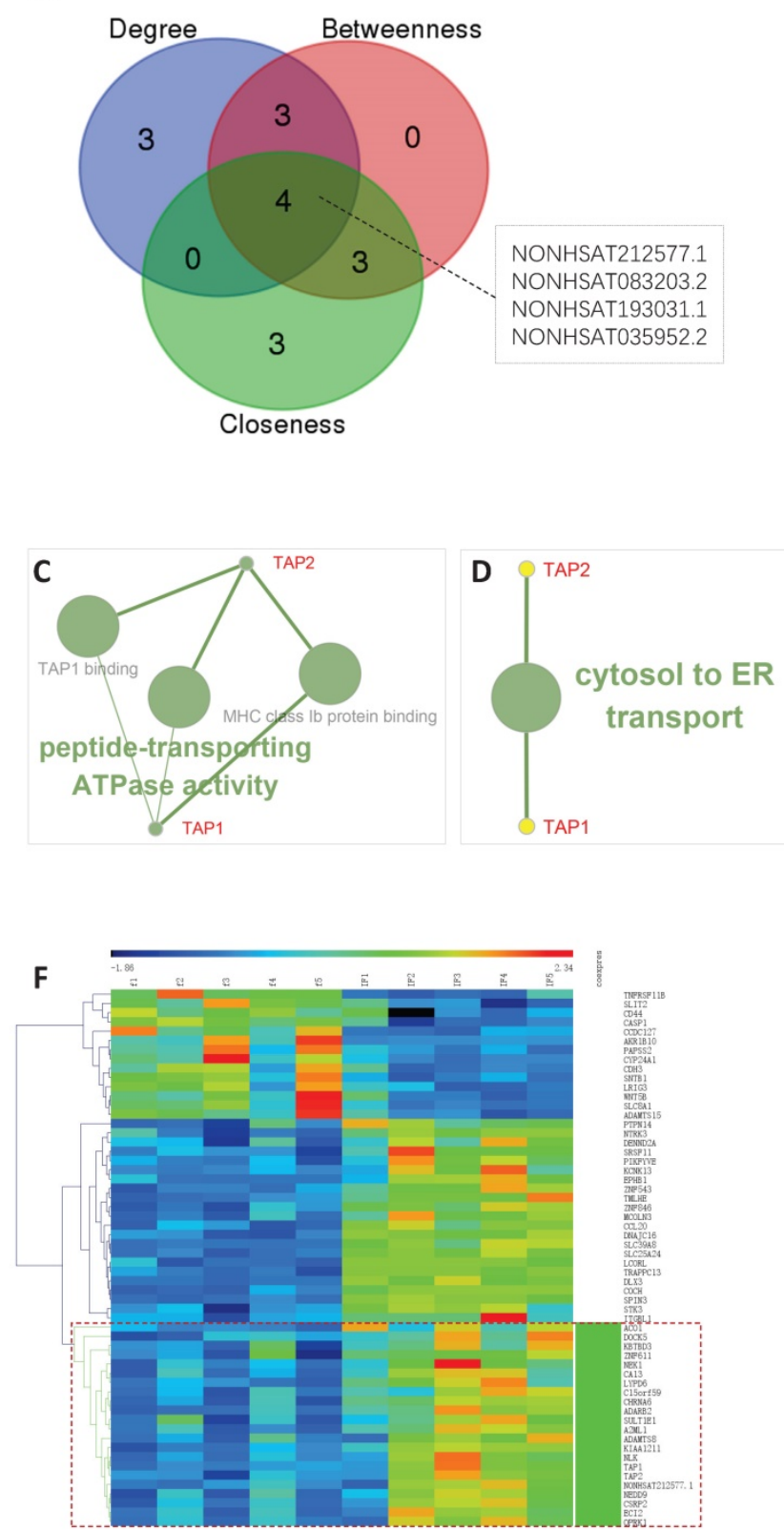

B

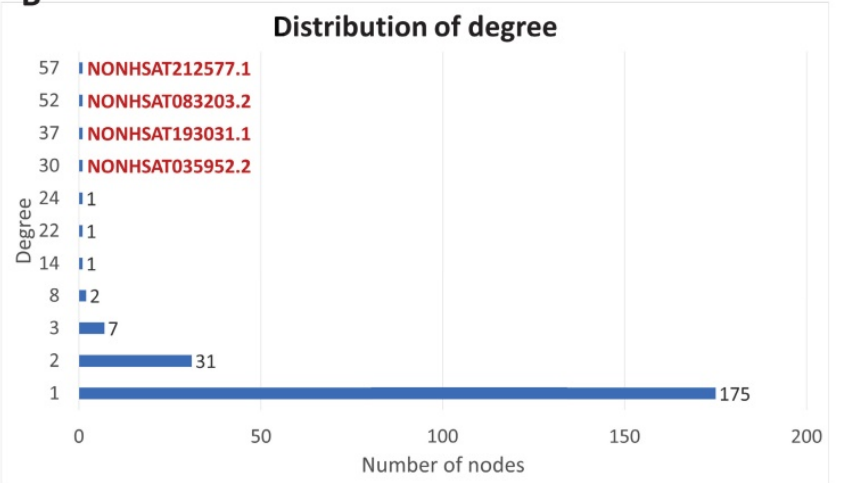

E
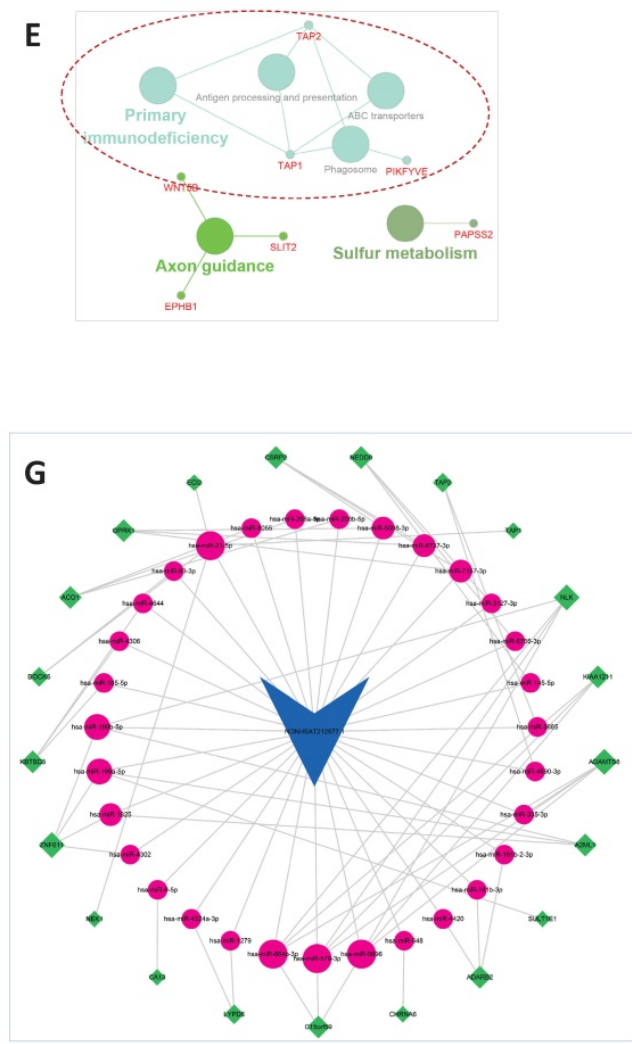

Figure 4. Topological characteristics of implantation failure related IncRNA-mRNA network (IFLMN) and ceRNA sub-network of NONHSAT212577.1. A. Topological characteristics of IFLMN. There are four genes existed simultaneously in top 10 lists of degree, betweenness, and closeness. B. Distribution of degree. The top four genes with highest degree were four IncRNAs. C. Molecular function enrichment of NONHSAT212577.1. D. Biological process enrichment of NONHSAT212577.1. E. KEGG enrichment of NONHSAT212577.1. F. The co-expression subnet of NONHSAT212577.1. The dotted lines emphasize the co-expressed genes. G. The ceRNA network of NONHSAT212577.1. The dotted lines emphasize the annotations that have been proved to work in endometrial receptivity.

KEGG analysis with $P \leq 0.05$ found six enriched KEGG pathways, as shown in Figure 4E and Table S2.

Molecular function was enriched in TAP1 binding, MHC class Ib protein binding, and peptide-transporting ATPase activity. Biological process was enriched in cytosol to ER transport. Both of these two pointed to TAP1 and TAP2 (Figure 4C and $4 \mathrm{D})$, indicating transcription of antigen peptide transporters TAP1 and TAP2 were crucial in endometrial receptivity. What's more, KEGG pathway analysis also pointed to TAP1 and TAP2 (Figure 4E), confirming their importance and emphasized the role of immunity in endometrial receptivity.

It is reported that TAP1 and TAP2 are up-regulated in pregnant animals [58]. TAP proteins may up-regulate VEGF-C and stimulate the capacity of natural killer cells to be non-cytotoxic, so TAP1 and TAP2 are involved in local immune suppression during pregnancy ${ }^{[59]}$. Silencing of TAP1 abolishes the VEGF-C participated immune protection [60]. IFNG significantly regulates TAP1, chemokines, enzymes, and TFs, induces uNK cell production of the chemokines, direct migration and invasion, and 
promote physiological angiogenesis in the placental bed [61]. The above indicates that TAP1 and TAP2 play a role in implantation.

Clustering of NONHSAT212577.1 and the 57 first neighbor mRNAs, the genes clustered together with the lncRNA were extracted. Then the linked miRNAs were added, and a new sub-network was constructed. In all, 21 mRNAs, 31 miRNAs, and 82 edges were included in the ceRNA sub-network of NONHSAT212577.1, as shown in Figure 4F and 4G.

For NONHSAT083203.2, 52 mRNAs were extracted from the IFLMN as the first mRNA neighbors. GO classification (Biological process) with $P \leq 0.05$ adjusted by Benjamini-Hochberg found 11 enriched GO terms, as shown in Figure 5A and Table S3. KEGG analysis with $P \leq 0.05$ found 8 enriched KEGG pathways, as shown in Figure 5B and Table S4.

Clustering of NONHSAT083203.2 and the 52 first neighbor mRNAs, the bundle of genes clustered together with the IncRNA were extract. Then the linked miRNAs were added, and a new sub-network was constructed. In all, 9 mRNAs, 20 miRNAs, and 105 edges were included in the ceRNA sub-network of NONHSAT083203.2, as shown in Figure 5C and $5 \mathrm{D}$.

For NONHSAT193031.1, 37 mRNAs were extracted from the IFLMN as the first mRNA neighbors. GO classification (Biological process) with $P \leq 0.05$ obtained 128 enriched GO terms, as shown in Figure 6A and Table S5. KEGG analysis with $P \leq 0.05$ found 8 enriched KEGG pathways, as shown in Figure 6B and Table S6.

Clustering of NONHSAT193031.1 and the 37 first neighbor mRNAs, the bundle of genes clustered together with the IncRNA were extract. Then the linked miRNAs were added, and a new sub-network was constructed. In all, 8 mRNAs, 11 miRNAs, and 27 edges were included in the ceRNA sub-network of NONHSAT193031.1, as shown in Figure 6C and 6D.

For NONHSAT035952.2, 30 mRNAs were extracted from the IFLMN as the first mRNA neighbors. GO classification (Biological process) with $P \leq 0.05$ obtained 43 enriched GO terms, as shown in Figure 7A and Table S7. KEGG analysis with $P \leq 0.05$ found 4 enriched KEGG pathways, as shown in Figure 7B and Table S8.
A

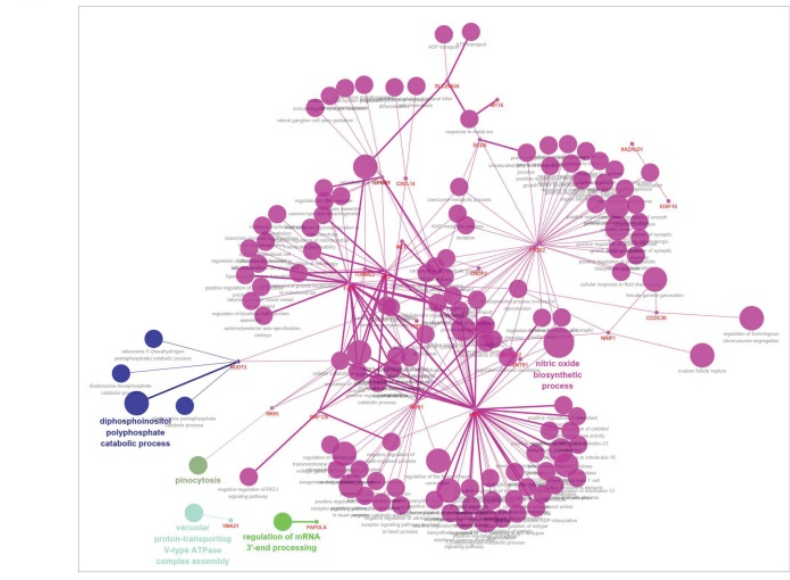

C

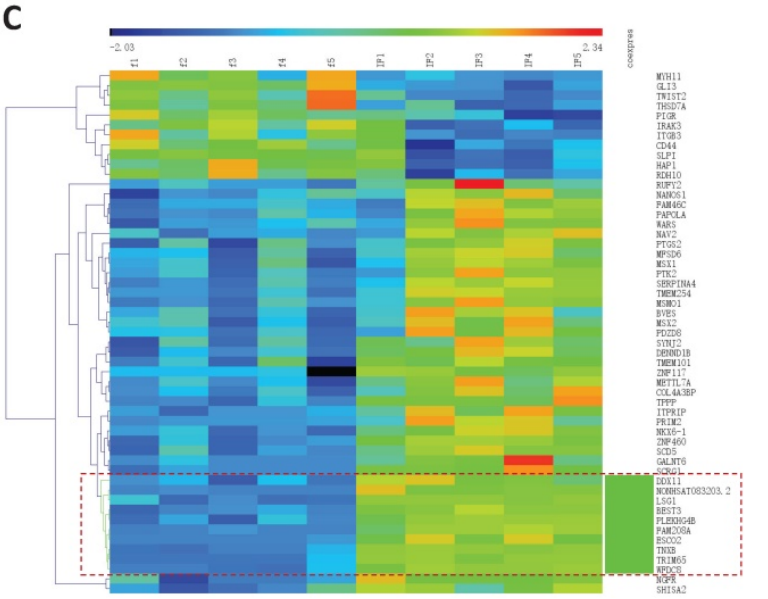

B

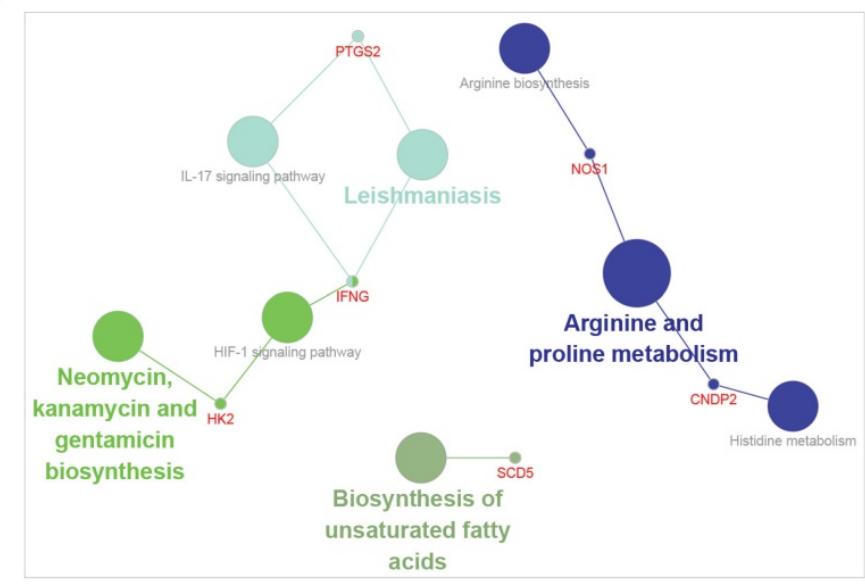

D

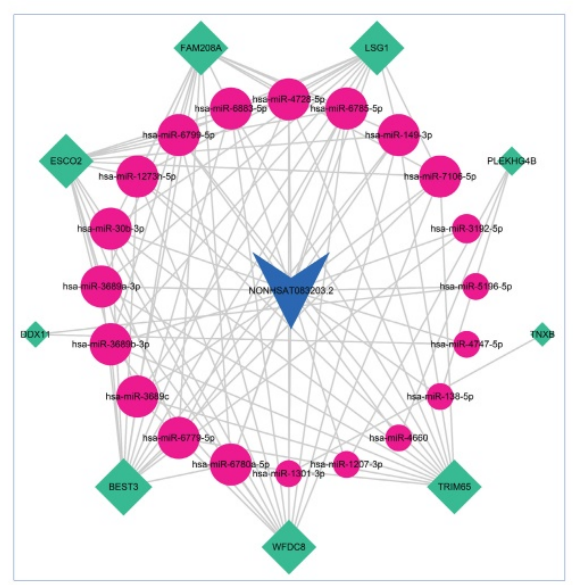

Figure 5. The ceRNA sub-network of NONHSAT083203.2. A. Biological process enrichment of NONHSAT083203.2. B. KEGG enrichment of NONHSAT083203.2. C. The co-expression subnet of NONHSAT083203.2. The dotted lines emphasize the co-expressed genes. D. The ceRNA network of NONHSAT083203.2. 
A

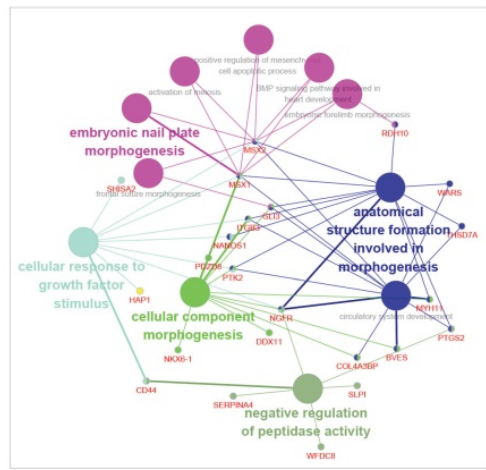

B

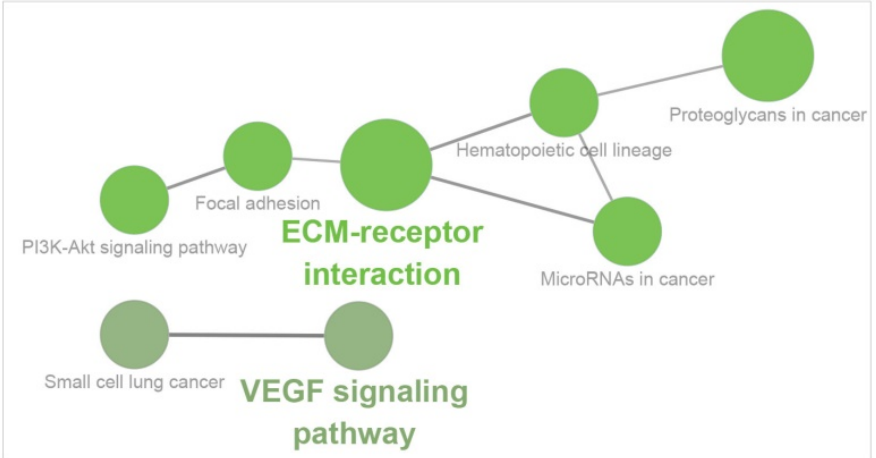

D

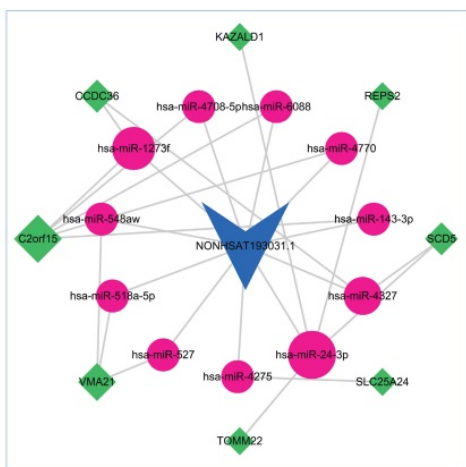

Figure 6. The ceRNA sub-network of NONHSAT193031.1. A. Biological process enrichment of NONHSAT193031.1. B. KEGG enrichment of NONHSAT193031.1. C. The co-expression subnet of NONHSAT193031.1. The dotted lines emphasize the co-expressed genes. D. The ceRNA network of NONHSAT193031.1.

A

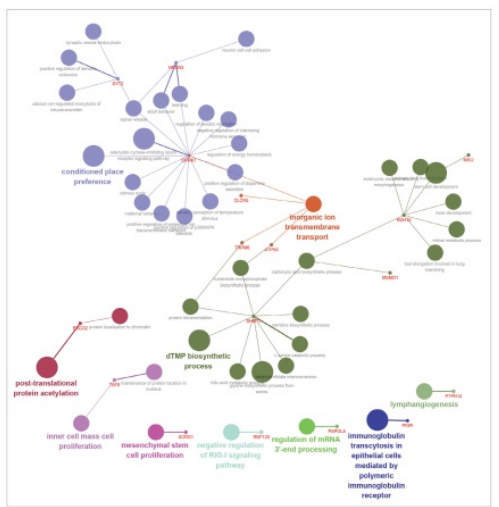

B

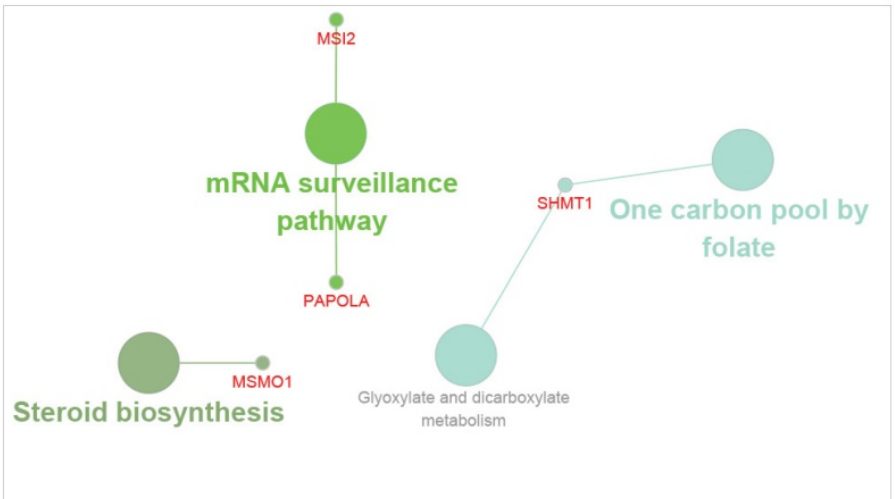

C

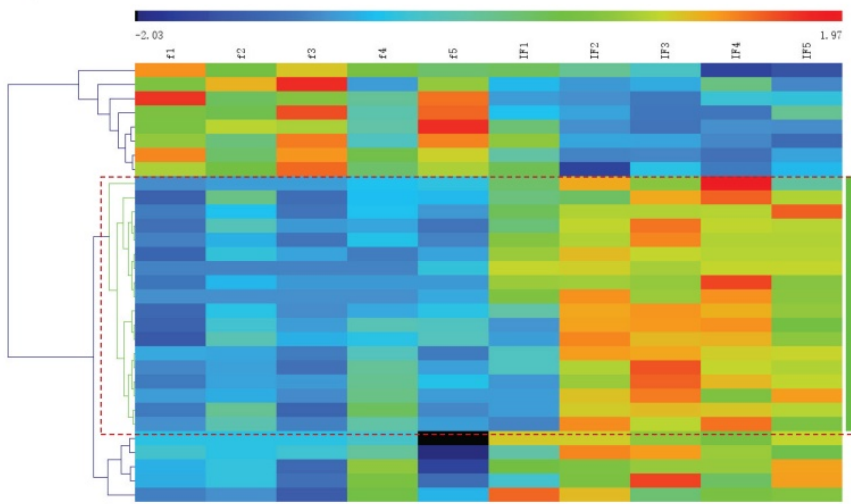

D

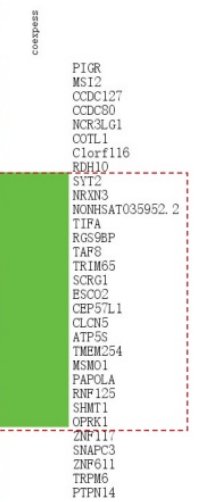

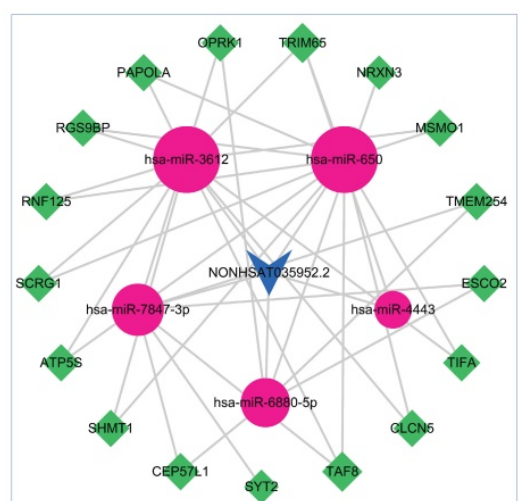

Figure 7. The ceRNA sub-network of NONHSAT035952.2. A. Biological process enrichment of NONHSAT035952.2. B. KEGG enrichment of NONHSAT035952.2. C. The co-expression subnet of NONHSAT035952.2. The dotted lines emphasize the co-expressed genes. D. The ceRNA network of NONHSAT035952.2. 
Clustering of NONHSAT035952.2 and the 30 first neighbor mRNAs, the bundle of genes clustered together with the lncRNA were extract, then the linked miRNAs were added, and a new sub-network was constructed. In all, 17 mRNAs, 5 miRNAs, and 39 edges were included in the ceRNA sub-network of NONHSAT035952.2, as shown in Figure 7C and 7D.

\section{Co-expression modules.}

The lncRNAs and mRNAs included in the IFLMN was further selected with Pearson correlation analysis. The lncRNA-mRNA pairs with correlation coefficient $>0.632$ and $P<0.05$ was used for bidirectional clustering. There were 10 lncRNAs, 163 mRNAs, and 192 lncRNA-mRNA pairs selected for further bidirectional clustering.

As shown in Figure 8A, two co-expression modules were found. Module 1 included 3 lncRNAs and 29 mRNAs, and then 50 miRNAs were extracted from the global triple network. At last, 246 edges were included in the module 1 ceRNA sub-network (Figure 8B, Table S9). Module 2 included 2 lncRNAs and 13 mRNAs. Six miRNAs were extracted from the global triple network. At last, 28 edges were included in the module 2 ceRNA sub-network (Figure 8C, Table S9).

For Module 1, GO classification (Molecular function) with $P \leq 0.05$ obtained 35 enriched GO terms, as shown in Figure 8D. KEGG analysis with $P \leq$ 0.05 found three enriched KEGG pathways, as shown in Figure 8E.

Phosphatidylinositol 3 kinase (PI3K)-Akt signaling pathway was enriched in Module 1 (Figure $8 \mathrm{E})$. It is reported that embryo implantation is disturbed when PI3K-Akt signaling pathway is blocked [62]. Cell motility and invasion are crucial events for endometrial remodeling during embryo implantation. Steroid hormones and growth factors can activate PI3K-Akt signaling pathway and stimulate migration of endometrial stromal cells [63]. TNXB was involved in PI3K-Akt pathway. As shown in Figure 8B, hsa-miR-1301-3p, as link between NONHSAT083203.2 and TNXB, may work as sponge of this interaction to involve in endometrial receptivity.

Apoptosis signaling pathway was also enriched in Module 1 (Figure 8E). The proliferation and apoptosis of the endometrium is the foundation of the preparation for the WOI [64]. High estradiol concentration can induce the apoptosis of endometrial glandular cells and impairs endometrial receptivity, leading to adverse reproductive outcome in IVF cycles [65], which explains the reason why pregnancy rate of frozen cycle is better than that of fresh cycle. NGFR was involved in apoptosis pathway. As shown in Figure 8B, hsa-miR-6785-5p, hsa-miR-6883-5p, hsa-miR-4728-5p, hsa-miR-149-3p, hsa-miR-4747-5p, hsa-miR-5196-5p, hsa-miR-7106-5p, and hsa-miR-3202 linked NONHSAT083203.2/NONHSAT025064.2 and NGFR, and they may work as sponges of these interactions to involve in endometrial receptivity.

For Module 2, GO classification (Molecular function) with $P \leq 0.05$ obtained 12 enriched GO terms, as shown in Figure 8F. KEGG analysis with $P \leq$ 0.05 found six enriched KEGG pathways, as shown in Figure 8G.

Steroid biosynthesis pathway was enriched in Module 2 (Figure 8G). As we emphasized above, steroid biosynthesis pathway may play an important role in endometrial receptivity. MSMO1 was involved in steroid biosynthesis pathway. As shown in Figure 8C, hsa-miR-6880-5p, hsa-miR-650, and hsa-miR-3612, as links between NONHSAT053761.2 /NONHSAT03 5952.2 and MSMO1, may work as sponges of these interactions to involve in endometrial receptivity.

\section{Validation of IncRNAs expression of endometrium}

There were no significant differences between RIF/RM group and control group in age, BMI (body mass index), endometrial pattern, and endometrial thickness $(P>0.05)$. The identified candidate lncRNAs were assayed by real-time PCR on a sample set of 8 RIF/RM patients and 8 controls. As shown in Figure 9, the expression levels of NONHSAT083203.2, NONHSAT212577.1, NONHSAT035952.2, NONHSA T193031.1, NONHSAT053761.2, and NONHSAT025 064.2 in RIF/RM patients were significantly higher than those in controls $(P<0.01)$. The increased amplitudes range from 4.17 to 38.68 folds, which is obviously a substantial elevation.

Our previous bioinformatics analysis discovered that these six lncRNAs were upregulated in IF patients and might predict endometrial receptivity. Furthermore, we investigated the expression of these lncRNAs in endometrium and validated their upregulation in RIF patients. It is reported that in patients with better endometrial receptivity, the expression of ST7-OT3 was down-regulated, which supported out results [66]. Considering their role in IFLMN and their functional enrichment, it will be meaningful to explore their upstream regulation mechanism and their downstream effector mechanism. 
A

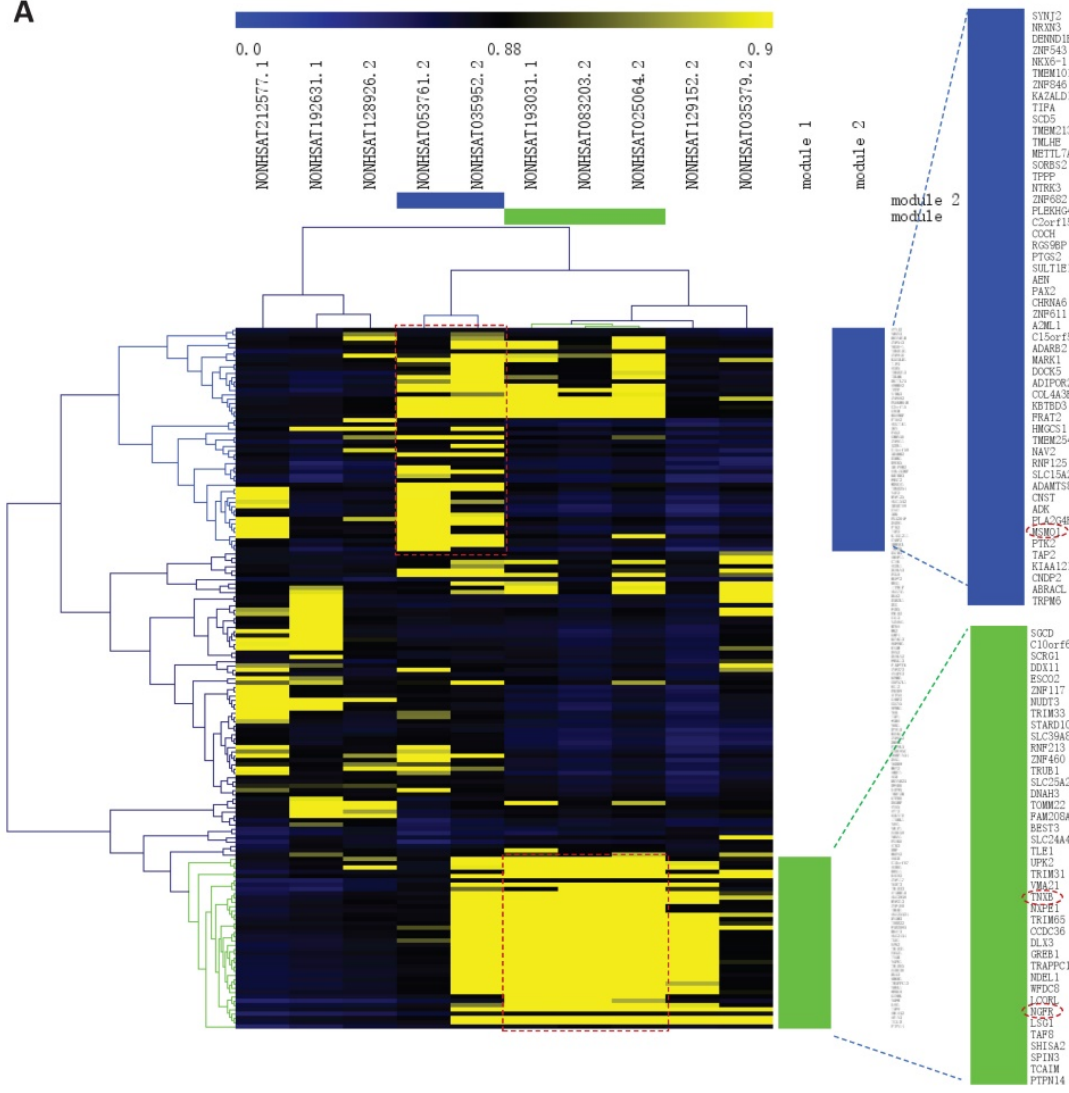

B

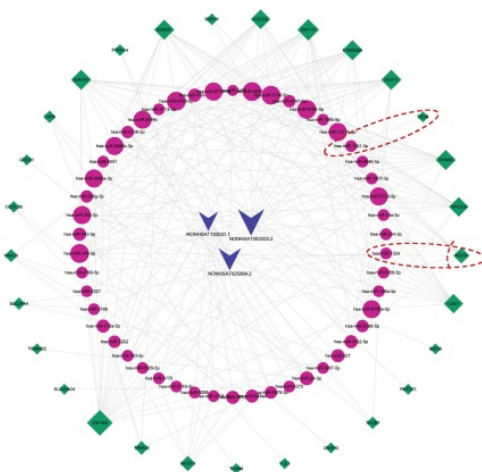

C

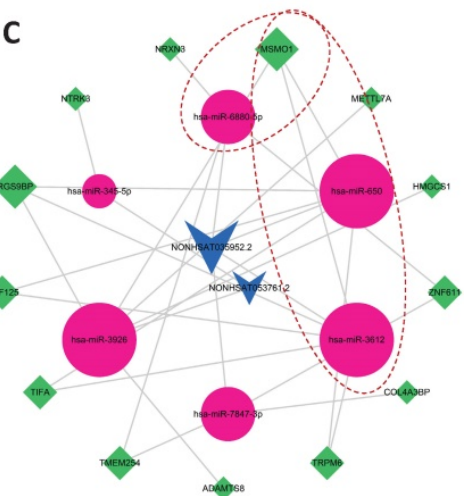

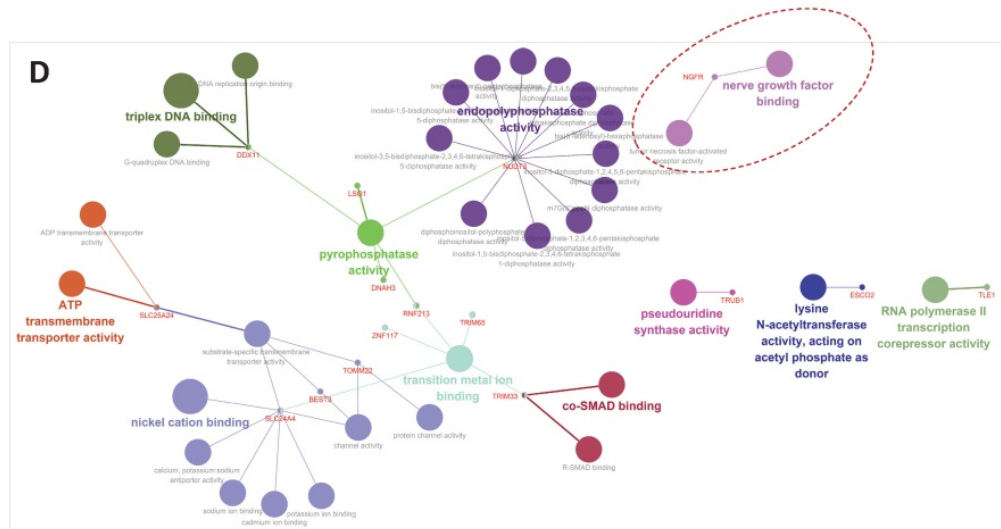

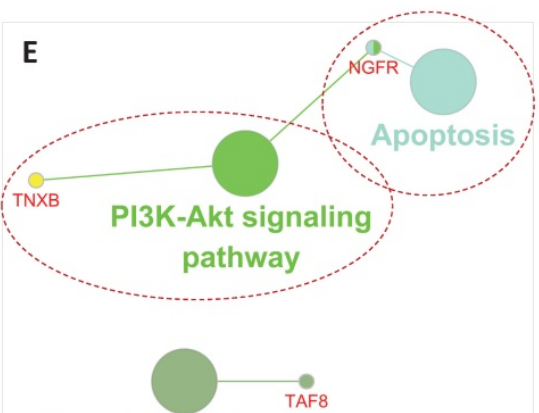

Basal transcription

factors

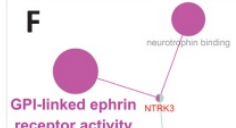

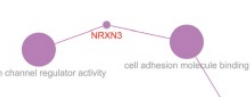

ADMirses

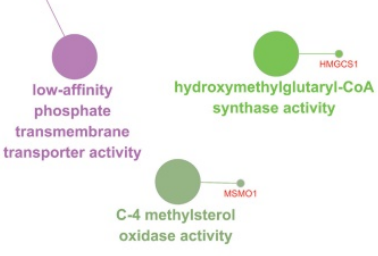

binding
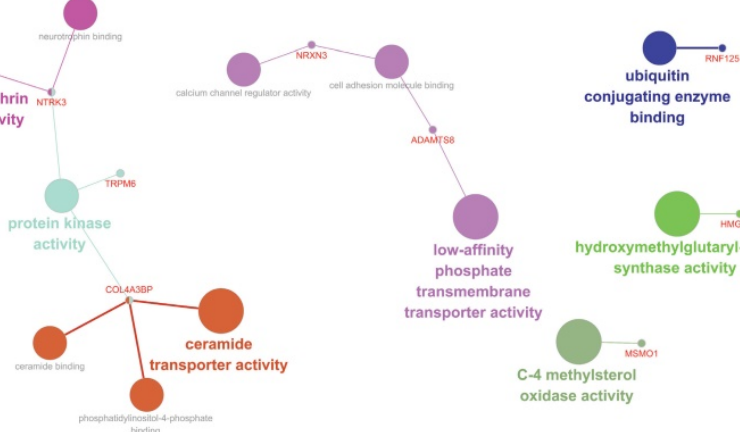

G

Synthesis and

degradation of

ketone bodies HMGCS1

Figure 8. The co-expression networks in implantation failure related IncRNA-mRNA network (IFLMN). A. The bidirectional clustering of IFLMN. The genes with green bar were included in Module 1 and the genes with blue bar were included in Module 2. B. Module 1 of ceRNA networks. Module 1 included 3 IncRNAs and 29 mRNAs. C. Module 2 of ceRNA networks. Module 2 included 2 IncRNAs and 13 mRNAs. D. GO classification of Module 1. E. KEGG pathway of Module 1. F. GO classification of Module 2. G. KEGG pathway of Module 2. 


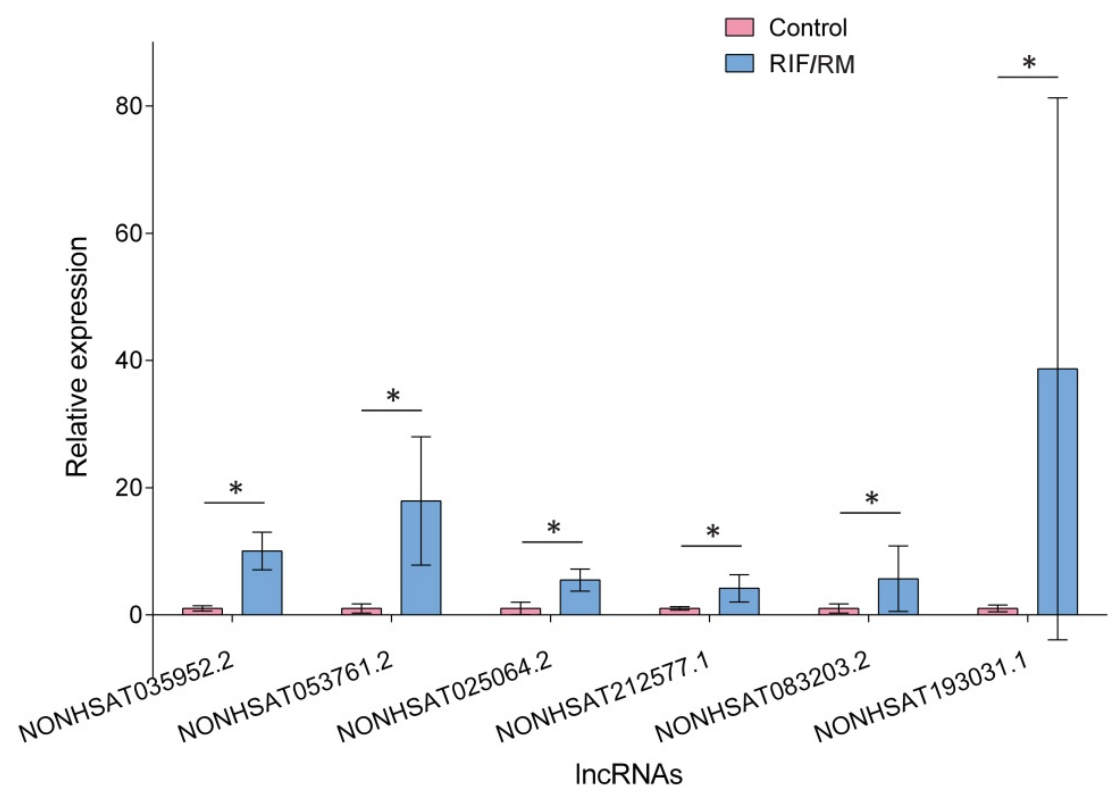

Figure 9. Expression of IncRNAs in endometrium. The six key IncRNAs were assayed by real-time PCR on a sample set of 8 RIF patients and 8 controls. The expression levels of IncRNAs in RIF increased significantly compared to fertile controls. * $P<0.05$.

A gene expression microarray study of endometrium in 2016 identified a signature containing 303 genes predictive of RIF. Further validation in independent samples showed that the gene signature predicted RIF with $100 \%$ positive predictive value (PPV). Among them, there were 11 lncRNAs identified in NONCODE database, including NONHSAT007100.2, NONMMUT115606.1, NONHSAT076715.2, NONHSAT030758.2, NONHSA T169502.1, NONHSAT133392.2, NONHSAT084833.2, NONHSAT198142.1, NONHSAT131185.2, NONHSA T073810.2, NONHSAT039454.2 [68]. A lncRNA and mRNA microarray study of endometrium in 2017 detected 197 dysregulated lncRNAs in RIF endometrium compared with the control group. Real-time PCR validated the expression of ENSG00000267194.1 (p7482), XLOC_005748 (p23230), XLOC_004399 (p22979), ENSG00000224259.1 (p1238), ENSG00000261645.1 (p2900), ENSG00000267549.1 p8859), and ENSG00000229385.1 (p9212) were elevated and the expression of ENSG00000259716.1 (p10433) was decreased [67]. These results indicate that lncRNA expression of RIF endometrium was different from that of normal receptive endometrium, suggesting that lncRNAs may regulate endometrial receptivity. However, the key lncRNAs in the present study were not in accordance with the lncRNAs identified in these two studies. The reason might be as follows: ethnic disparities, difference microarray platforms, and different bioinformatics methodology.

\section{Conclusion}

In the present study, we constructed the IFLMN, identified six key lncRNAs in IFLMN through topological analysis, clustering and co-expression analyses, and validated their increased expression in endometrium of RIF/RM patients. NONHSAT 083203.2 (CECR3), NONHSAT212577.1 (ST7-OT3), NONHSAT035952.2 (DHRS4-AS1), NONHSAT1930 31.1 (C22orf34), NONHSAT053761.2 (RAMP2-AS1), and NONHSAT025064.2 (PNCT_HSA157732), together with their ceRNA sub-networks, may be involved in immunological activity, growth factor binding, vascular proliferation, apoptosis, and steroid biosynthesis in uterus and prepare endometrium for embryo implantation. These lncRNAs might be regulated by transcription factors that involved in sex steroid functions, such as PRA and PRB. Since some lncRNAs maintain stable in peripheral blood and are protected from endogenous RNases, lncRNAs can function as biomarkers for detection and diagnosis. These six lncRNA emerged as promising candidates to predict endometrial receptivity since their role in IFLMN and their functional enrichment. Further studies are needed to validate the above lncRNAs in uterine luminal fluid and peripheral blood, and they may be developed into predictive biomarkers for endometrial receptivity.

\section{Supplementary Material}

Supplementary figure and tables.

http://www.ijbs.com/v14p1361s1.pdf

\section{Acknowledgements}

This work was financially supported by the Province Zhejiang Provincial Natural Science Foundation (No. LY17H040005 to C. F.), National Natural Science Foundation of China (No. 81701461 to 
P.-P. L., 81671487 to M. J.), Medical and Health Science and Technology Plan of Zhejiang Province (No. 2017KY078 to C. F., 2017KY501 to J.-M. S., No. 2018251579 to P.-P. L.), Zhejiang Provincial Science and Technology Plan of Traditional Chinese Medicine (No. 2017ZQ013 to J.-M. S.), and Science and Technology Project of the competent department (No. LY17H040005 to P.-P. L.).

\section{Author Contributions}

C.F. and L.F. designed and supervised the experiments; P.-P. L., M. J. and L.-Q. W. downloaded, analyzed, and discussed the data; C. F., P.-P. L., J.-M. S. and J.-P. R. collected samples and performed real-time PCR; C. F. and J.-M. S. drafted the manuscript.

\section{Competing Interests}

The authors have declared that no competing interest exists.

\section{References}

[1] Chang J, Boulet SL, Jeng G, Flowers L, Kissin DM. Outcomes of in vitro fertilization with preimplantation genetic diagnosis: an analysis of the United States Assisted Reproductive Technology Surveillance Data, 2011-2012. Fertil Steril. 2016. 105(2): 394-400

[2] Rodriguez-Purata J, Lee J, Whitehouse M, et al. Reproductive outcome is optimized by genomic embryo screening, vitrification, and subsequent transfer into a prepared synchronous endometrium. J Assist Reprod Genet. 2016. 33(3): 401-412.

[3] Hatirnaz S, Ozer A, Hatirnaz E, et al. Pre-implantation genetic screening among women experiencing recurrent failure of in vitro fertilization. Int J Gynaecol Obstet. 2017. 137(3): 314-318.

[4] Palmieri G, Paliogiannis P, Sini MC, et al. Long non-coding RNA CASC2 in human cancer. Crit Rev Oncol Hematol. 2017. 111: 31-38.

[5] Li W, Li H, Zhang L, et al. Long non-coding RNA LINC00672 contributes to p53 protein-mediated gene suppression and promotes endometrial cancer chemosensitivity. J Biol Chem. 2017. 292(14): 5801-5813.

[6] Liu L, Chen X, Zhang Y, Hu Y, Shen X, Zhu W. Long non-coding RNA TUG1 promotes endometrial cancer development via inhibiting miR-299 and miR-34a-5p. Oncotarget. 2017. 8(19): 31386-31394.

[7] Shang C, Lang B, Ao CN, Meng L. Long non-coding RNA tumor suppressor candidate 7 advances chemotherapy sensitivity of endometrial carcinoma through targeted silencing of miR-23b. Tumour Biol. 2017. 39(6): 1010428317707883

[8] Men Y, Fan Y, Shen Y, Lu L, Kallen AN. The steroidogenic acute regulatory protein (StAR) is regulated by the H19/let-7 axis. Endocrinology. 2017. 158(2): 402-409.

[9] Zhou C, Zhang T, Liu F, et al. The differential expression of mRNAs and long noncoding RNAs between ectopic and eutopic endometria provides new insights into adenomyosis. Mol Biosyst. 2016. 12(2): 362-370.

[10] Wang Y, Li Y, Yang Z, Liu K, Wang D. Genome-wide microarray analysis of long non-coding RNAs in eutopic secretory endometrium with endometriosis. Cell Physiol Biochem. 2015. 37(6): 2231-2245.

[11] Wang Y, Hu T, Wu L, Liu X, Xue S, Lei M. Identification of non-coding and coding RNAs in porcine endometrium. Genomics. 2017. 109(1): 43-50.

[12] Zeng H, Fan X, Liu N. Expression of H19 imprinted gene in patients with repeated implantation failure during the window of implantation. Arch Gynecol Obstet. 2017. 296(4): 835-839.

[13] Li J, Cao Y, Xu X, et al. Increased New lncRNA-mRNA Gene Pair Levels in Human Cumulus Cells Correlate With Oocyte Maturation and Embryo Development. Reprod Sci. 2015. 22(8): 1008-1014

[14] Salmena L, Poliseno L, Tay Y, Kats L, Pandolfi PP. A ceRNA hypothesis: the Rosetta Stone of a hidden RNA language. Cell. 2011. 146(3): 353-358.

[15] Chen J, Xu J, Li Y, et al. Competing endogenous RNA network analysis identifies critical genes among the different breast cancer subtypes. Oncotarget. 2017. 8(6): 10171-10184.

[16] Zhao L, Liu B. Identification of potential prognostic ceRNA module biomarkers in patients with pancreatic adenocarcinoma. Oncotarget. 2017. 8(55): 94493-94504.

[17] Jin X, Guan $Y$, Sheng H, Liu Y. Crosstalk in competing endogenous RNA network reveals the complex molecular mechanism underlying lung cancer. Oncotarget. 2017. 8(53): 91270-91280.
[18] Zhang Y, Kang R, Liu W, et al. Identification and analysis of p53-mediated competing endogenous rna network in human hepatocellular carcinoma. Int J Biol Sci. 2017. 13(9): 1213-1221.

[19] Wang JB, Liu FH, Chen JH, et al. Identifying survival-associated modules from the dysregulated triplet network in glioblastoma multiforme. J Cancer Res Clin Oncol. 2017. 143(4): 661-671.

[20] Zhang M, Du X. Noncoding RNAs in gastric cancer: Research progress and prospects. World J Gastroenterol. 2016. 22(29): 6610-6618.

[21] Miao YR, Liu W, Zhang Q, Guo AY. IncRNASNP2: an updated database of functional SNPs and mutations in human and mouse lncRNAs. Nucleic Acids Res. 2017. 46(D1): D276-D280.

[22] Chou CH, Shrestha S, Yang CD, et al. miRTarBase update 2018: a resource for experimentally validated microRNA-target interactions. Nucleic Acids Res. 2017. 46(D1): D296-D302.

[23] Lédée N, Munaut C, Aubert J, et al. Specific and extensive endometrial deregulation is present before conception in IVF/ICSI repeated implantation failures (IF) or recurrent miscarriages. J Pathol. 2011. 225(4): 554-564.

[24] Harbron C, Chang KM, South MC. RefPlus: an R package extending the RMA Algorithm. Bioinformatics. 2007. 23(18): 2493-2494.

[25] Ritchie ME, Phipson B, Wu D, et al. limma powers differential expression analyses for RNA-sequencing and microarray studies. Nucleic Acids Res. 2015. 43(7): e47.

[26] Song C, Zhang J, Liu Y, et al. Construction and analysis of cardiac hypertrophy-associated lncRNA-mRNA network based on competitive endogenous RNA reveal functional IncRNAs in cardiac hypertrophy. Oncotarget. 2016. 7(10): 10827-10840.

[27] Livak KJ, Schmittgen TD. Analysis of relative gene expression data using real-time quantitative PCR and the 2(-Delta Delta C(T)) Method. Methods. 2001; 25: 402-408.

[28] Enciso M, Carrascosa JP, Sarasa J, et al. Development of a new comprehensive and reliable endometrial receptivity map (ER Map/ER Grade) based on RT-qPCR gene expression analysis. Hum Reprod. 2018. 33(2):220-228.

[29] Giudice LC. Microarray expression profiling reveals candidate genes for human uterine receptivity. Am J Pharmacogenomics. 2004. 4(5): 299-312.

[30] Dambaeva SV, Durning M, Rozner AE, Golos TG. Immunophenotype and cytokine profiles of rhesus monkey CD56bright and CD56dim decidual natural killer cells. Biol Reprod. 2012. 86(1): 1-10.

[31] Murphy SP, Tayade C, Ashkar AA, Hatta K, Zhang J, Croy BA. Interferon gamma in successful pregnancies. Biol Reprod. 2009. 80(5): 848-859.

[32] Kitaya K, Yasuo T, Yamaguchi T, Fushiki S, Honjo H. Genes regulated by interferon-gamma in human uterine microvascular endothelial cells. Int J Mol Med. 2007. 20(5): 689-697.

[33] Rodriguez AM, Mallet V, Lenfant F, et al. Interferon-gamma rescues HLA class Ia cell surface expression in term villous trophoblast cells by inducing synthesis of TAP proteins. Eur J Immunol. 1997. 27(1): 45-54.

[34] Karizbodagh MP, Rashidi B, Sahebkar A, Masoudifar A, Mirzaei H. Implantation Window and Angiogenesis. J Cell Biochem. 2017. 118(12): 4141-4151.

[35] Sirohi VK, Gupta K, Kumar R, Shukla V, Dwivedi A. Selective estrogen receptor modulator Ormeloxifene suppresses embryo implantation via inducing miR-140 and targeting insulin-like growth factor 1 receptor in rat uterus. J Steroid Biochem Mol Biol. 2018. 178: 272-282.

[36] Zhang L, Liu X, Liu J, et al. MiR-26a promoted endometrial epithelium cells (EECs) proliferation and induced stromal cells (ESCs) apoptosis via the PTEN-PI3K/AKT pathway in dairy goats. J Cell Physiol. 2017. 233(6):4688-4706

[37] Liu H, Chen Y, Yan F, et al. Ultrasound molecular imaging of vascular endothelial growth factor receptor 2 expression for endometrial receptivity evaluation. Theranostics. 2015. 5(2): 206-217.

[38] Salker MS, Singh Y, RRP D, et al. LEFTY2 inhibits endometrial receptivity by downregulating Orai1 expression and store-operated Ca2+ entry. J Mol Med (Berl). 2017. 96(2):173-182.

[39] Gibson DA, McInnes KJ, Critchley HO, Saunders PT. Endometrial Intracrinology--generation of an estrogen-dominated microenvironment in the secretory phase of women. J Clin Endocrinol Metab. 2013. 98(11): E1802-E1806.

[40] Tseng L, Mazella J, Mann WJ, Chumas J. Estrogen synthesis in normal and malignant human endometrium. J Clin Endocrinol Metab. 1982. 55(5): 1029-1031.

[41] Hannan NJ, Paiva P, Meehan KL, Rombauts LJ, Gardner DK, Salamonsen LA. Analysis of fertility-related soluble mediators in human uterine fluid identifies VEGF as a key regulator of embryo implantation. Endocrinology. 2011. 152(12): 4948-4956.

[42] Gupta K, Sirohi VK, Kumari S, et al. Sorcin is involved during embryo implantation via activating VEGF/PI3K/Akt pathway in mice. J Mol Endocrinol. 2017. 60(2):119-132.

[43] Demir R, Yaba A, Huppertz B. Vasculogenesis and angiogenesis in the endometrium during menstrual cycle and implantation. Acta Histochem. 2010. 112(3): 203-214

[44] Jinno M, Ozaki T, Iwashita M, Nakamura Y, Kudo A, Hirano H. Measurement of endometrial tissue blood flow: a novel way to assess uterine receptivity for implantation. Fertil Steril. 2001. 76(6): 1168-1174.

[45] Chen X, Jin X, Liu L, et al. Differential expression of vascular endothelial growth factor angiogenic factors in different endometrial compartments in women who have an elevated progesterone level before oocyte retrieval, 
during in vitro fertilization-embryo transfer treatment. Fertil Steril. 2015. 104(4): 1030-1036.

[46] Bhurke AS, Bagchi IC, Bagchi MK. Progesterone-Regulated Endometrial Factors Controlling Implantation. Am J Reprod Immunol. 2016. 75(3): 237-245.

[47] Mantena SR, Kannan A, Cheon YP, Li Q, Johnson PF, Bagchi IC, et al. C/EBPbeta is a critical mediator of steroid hormone-regulated cell proliferation and differentiation in the uterine epithelium and stroma. Proc Natl Acad Sci U S A. 2006; 103: 1870-1875.

[48] Yang S, Fang Z, Suzuki T, et al. Regulation of aromatase P450 expression in endometriotic and endometrial stromal cells by CCAAT/enhancer binding proteins (C/EBPs): decreased C/EBPbeta in endometriosis is associated with overexpression of aromatase. J Clin Endocrinol Metab. 2002. 87(5): 2336-2345.

[49] Tamura I, Shirafuta Y, Jozaki K, et al. Novel function of a transcription factor WT1 in regulating decidualization in human endometrial stromal cells and its molecular mechanism. Endocrinology. 2017. 158(10): 3696-3707.

[50] Wang W, Li Q, Bagchi IC, Bagchi MK. The CCAAT/enhancer binding protein beta is a critical regulator of steroid-induced mitotic expansion of uterine stromal cells during decidualization. Endocrinology. 2010; 151: 3929-3940.

[51] Lessey BA, Palomino WA, Apparao KB, Young SL, Lininger RA. Estrogen receptor-alpha (ER-alpha) and defects in uterine receptivity in women. Reprod Biol Endocrinol. 2006; 4 Suppl 1: S9.

[52] El-Hamarneh T, Hey-Cunningham AJ, Berbic M, Al-Jefout M, Fraser IS, Black K. Cellular immune environment in endometrial polyps. Fertil Steril. 2013. 100(5): 1364-1372.

[53] Jiang R, Yan G, Xing J, et al. Abnormal ratio of CD57+ cells to CD56+ cells in women with recurrent implantation failure. Am J Reprod Immunol. 2017. $78(5)$.

[54] Bourdiec A, Ahmad SF, Lachhab A, Akoum A. Regulation of inflammatory and angiogenesis mediators in a functional model of decidualized endometrial stromal cells. Reprod Biomed Online. 2016. 32(1): 85-95.

[55] Bourdiec A, Martel V, Akoum A. Synchronous regulation of the determinants of endometrial receptivity to interleukin 1 at key stages of early embryo implantation in vivo. Fertil Steril. 2014. 101(4): 1183-93.

[56] Shi C, Han HJ, Fan LJ, et al. Diverse endometrial mRNA signatures during the window of implantation in patients with repeated implantation failure. Hum Fertil (Camb). 2017: 1-12.

[57] Zhang L, An XP, Liu XR, et al. Characterization of the transcriptional complexity of the receptive and pre-receptive endometria of dairy goats. Sci Rep. 2015. 5: 14244

[58] Walker CG, Meier S, Littlejohn MD, Lehnert K, Roche JR, Mitchell MD. Modulation of the maternal immune system by the pre-implantation embryo. BMC Genomics. 2010. 11: 474

[59] Kalkunte SS, Mselle TF, Norris WE, Wira CR, Sentman CL, Sharma S. Vascular endothelial growth factor $\mathrm{C}$ facilitates immune tolerance and endovascular activity of human uterine NK cells at the maternal-fetal interface. J Immunol. 2009. 182(7): 4085-4092

[60] Lash GE, Ansari T, Bischof P, et al. IFPA meeting 2008 workshops report. Placenta. 2009. 30 Suppl A: S4-14.

[61] Zhang J, Chen Z, Smith GN, Croy BA. Natural killer cell-triggered vascular transformation: maternal care before birth. Cell Mol Immunol. 2011. 8(1): 1-11.

[62] Liu L, Wang Y, Yu Q. The PI3K/Akt signaling pathway exerts effects on the implantation of mouse embryos by regulating the expression of RhoA. Int J Mol Med. 2014. 33(5): 1089-1096.

[63] Gentilini D, Busacca M, Di FS, Vignali M, Viganò P, Di BAM. PI3K/Akt and ERK1/2 signalling pathways are involved in endometrial cell migration induced by 17beta-estradiol and growth factors. Mol Hum Reprod. 2007. 13(5): 317-322.

[64] Lessey BA, Kim JJ. Endometrial receptivity in the eutopic endometrium of women with endometriosis: it is affected, and let me show you why. Fertil Steril. 2017. 108(1): 19-27.

[65] Chen SU, Chou CH, Chen MJ, Chen TH, Yang YS, Yang JH. Apoptotic effects of high estradiol concentrations on endometrial glandular cells. J Clin Endocrinol Metab. 2014. 99(6): E971-980.

[66] Chan K. Transcriptomic approach to study the effect of polypectomy on human endometrial receptivity. HKU Theses Online (HKUTO). 2014.

[67] Fan LJ, Han HJ, Guan J, Zhang XW, Cui QH, Shen H, et al. Aberrantly expressed long noncoding RNAs in recurrent implantation failure: A microarray related study. Syst Biol Reprod Med. 2017; 63: 269-278,

[68] Koot YE, van Hooff SR, Boomsma CM, van Leenen D, Groot KMJ, Goddijn M, et al. An endometrial gene expression signature accurately predicts recurrent implantation failure after IVF. Sci Rep. 2016; 6: 19411. 